\title{
Fourier, Gegenbauer and Jacobi Expansions for a Power-Law Fundamental Solution of the Polyharmonic Equation and Polyspherical Addition Theorems
}

\author{
Howard S. COHL \\ Applied and Computational Mathematics Division, National Institute of Standards \\ and Technology, Gaithersburg, MD, 20899-8910, USA \\ E-mail: howard.cohl@nist.gov
}

URL: http://hcohl.sdf .org

Received November 29, 2012, in final form May 28, 2013; Published online June 05, 2013

http://dx.doi.org/10.3842/SIGMA.2013.042

\begin{abstract}
We develop complex Jacobi, Gegenbauer and Chebyshev polynomial expansions for the kernels associated with power-law fundamental solutions of the polyharmonic equation on $d$-dimensional Euclidean space. From these series representations we derive Fourier expansions in certain rotationally-invariant coordinate systems and Gegenbauer polynomial expansions in Vilenkin's polyspherical coordinates. We compare both of these expansions to generate addition theorems for the azimuthal Fourier coefficients.
\end{abstract}

Key words: fundamental solutions; polyharmonic equation; Jacobi polynomials; Gegenbauer polynomials; Chebyshev polynomials; eigenfunction expansions; separation of variables; addition theorems

2010 Mathematics Subject Classification: 35A08; 31B30; 31C12; 33C05; 42A16

\section{Introduction}

We have developed a technique for constructing addition theorems for the azimuthal Fourier coefficients of fundamental solutions for linear homogeneous partial differential equations on $d$-dimensional isotropic Riemannian manifolds. For a fundamental solution, we construct azimuthal Fourier expansions and compare them with eigenfunction expansions in rotationallyinvariant coordinate systems. The construction of eigenfunction expansions for fundamental solutions in separable coordinate systems is in general non-trivial.

In connection with the Laplace operator, we have already constructed some of these addition theorems on $\mathbb{R}^{3}[5,10,11]$, where we have treated the spherical, cylindrical, oblate spheroidal, prolate spheroidal, parabolic, bispherical and toroidal coordinate systems. One may construct addition theorems in this manner in any rotationally-invariant coordinate system which yields solutions through separation of variables for the Laplace equation. In a similar setting, addition theorems may be generated for other inhomogeneous linear partial differential equations, such as for the Helmholtz, wave and heat equations in arbitrary dimensions. Furthermore an extension of this concept is possible when working with linear partial differential operators on Riemannian manifolds, such as for the Laplace-Beltrami operator (see $[9, \S 5.1]$ ). Once a Fourier expansion for a fundamental solution is obtained for a partial differential operator on a Riemannian manifold, one must construct eigenfunction expansions for a fundamental solution corresponding to that operator and identify those nested multi-summation and multi-integration eigenfunction expansions which correspond to the Fourier coefficients for that operator. 
In this paper, we apply this technique to generate addition theorems from eigenfunction expansions for a fundamental solution of the polyharmonic operator on $d$-dimensional Euclidean space $\mathbb{R}^{d}$ in Vilenkin-Kuznetsov-Smorodinskiǔ polyspherical coordinate systems [32, § 9.5], [34], [33, $\S 10.5]$ (hereafter Vilenkin). We have computed azimuthal Fourier expansions for a fundamental solution of this operator, as well as the corresponding eigenfunction expansions in polyspherical coordinates. In each case, the comparison of these two expansions yields new addition theorems for the azimuthal Fourier coefficients.

The main results of this paper are connected with closed-form expressions of hypergeometric orthogonal polynomial expansions for a power-law fundamental solution of the polyharmonic equation on $d$-dimensional Euclidean space. These expansions are the fundamental building blocks for algorithms to compute the solution of inhomogeneous linear polyharmonic boundary value problems through convolution with the source distribution. These problems are ubiquitous in physics and engineering and include elasticity, electrostatics, magnetostatics, quantum direct and exchange Coulomb interactions, Newtonian gravity, and potential fluid and heat flow, just to name a few. The expansions presented in this paper are crucial for obtaining analytic polyharmonic solutions and for numerical algorithms where expansions are needed for so-called "fast algorithms". Applications of generalized Hopf coordinates, one of the polyspherical coordinate systems we study in this paper include particle physics [24], quantum field theory [2] and cosmology [22]. Furthermore, expansions in hyperspherical coordinate systems have many applications including general atomic multibody theory (see [14, 23] and references therein).

From a global analytic partial differential equation perspective, the most important results of this paper are contained in Corollaries 2 and 4 . These formulae represent multipole and azimuthal Fourier decompositions for arbitrary powers of the Euclidean distance between two points. They can be used to analytically and numerically solve, in a rapidly convergent fashion, the inhomogeneous linear polyharmonic equation for isolated non-axisymmetric source distributions. Rapid convergence of the Fourier expansions is provided by the fact that for each azimuthal mode there corresponds an infinite number of meridional modes, which are all summed over in our expansions. Furthermore, if pure trigonometric azimuthal dependence exists for a particular source distribution in the inhomogeneous partial differential equation, then Corollary 4 provides a solution in a finite number of terms. In the case of an axisymmetric source distribution, the inhomogeneous polyharmonic solution is obtained from a single $m=0$ term in the expansion.

Corollary 2 is powerful in that it provides a mechanism for determining the multipole moments associated with unrestricted powers of the distance between two points. This is in contrast with the Gegenbauer generating function (which is generalized by Theorem 1 and Corollary 1) which provides with the addition theorem for hyperspherical harmonics (B.13) a multipole expansion for this kernel only for powers of the distance given by $\nu=2-d$, for $d=3,4,5, \ldots$.

From a special function theoretic perspective, the new results presented in this paper are Theorem 1 and Corollary 1. These series expansions represent fundamental generalizations of Heine's formula [27, (14.28.2)], Gegenbauer's generating function [27, (18.12.4)], and Heine's reciprocal square root identity $[8,(3.11)]$. Formula (A.14) which provides a connection between the symmetric Jacobi function of the second kind and the associated Legendre function of the second kind, is also interesting. As far as the author is aware, this has not previously appeared in the literature. The addition theorems for associated Legendre functions given by Theorems 2 and 3, and their Corollaries 5, 6, 7, also appear to be new. This paper only uses eigenfunction expansions for a power-law fundamental solution of the polyharmonic equation in Vilenkin's polyspherical coordinates to obtain new addition theorems for the azimuthal Fourier coefficients. We have only treated two different types of Vilenkin's polyspherical coordinates. In higher dimensions, many more types may be considered. The azimuthal Fourier expansion presented in this paper, Corollary 4, can be used to provide new addition theorems for the azimuthal Fourier coefficients in every rotationally-invariant coordinate system which separates 
the polyharmonic equation on $\mathbb{R}^{d}$. These rotationally-invariant coordinate systems include those of cylindrical, parabolic, and cyclidic type.

In this paper, we take advantage of previously derived closed-form expressions for the separated eigenfunctions in Vilenkin's polyspherical coordinates (found in [19,33] and elsewhere) to derive addition theorems from a power-law fundamental solution of the polyharmonic operator in Euclidean space $\mathbb{R}^{d}$. These addition theorems separate the complicated geometrically-relevant quantity (the azimuthal Fourier coefficients) into functions of the individual variables in the problem. We study all dimensions $d \geq 3$ with an emphasis on the simplicity/explicitness of the low-dimensional examples.

This paper is organized as follows. In Section 2, we describe the kernels associated with a fundamental solution of the polyharmonic equation on Euclidean space $\mathbb{R}^{d}$ for $d \geq 2$ and introduce rotationally-invariant coordinate systems. In Section 3, we prove several new theorems associated with Jacobi, Gegenbauer, and Chebyshev polynomial expansions for the kernels associated with power-law fundamental solutions of the polyharmonic equation on $\mathbb{R}^{d}$. In Section 4 , we derive and discuss new addition theorems in Vilenkin's polyspherical coordinates for the azimuthal Fourier coefficients of a fundamental solution for the polyharmonic equation on $\mathbb{R}^{d}$. In Appendix A, we summarize the definitions and properties of the special functions and orthogonal polynomials that we use. In Appendix B, we review Vilenkin's polyspherical coordinates and the corresponding normalized hyperspherical harmonics.

Throughout this paper we rely on the following definitions. Let $a_{1}, a_{2}, a_{3}, \ldots \in \mathbb{C}$, with $\mathbb{C}$ being the set of complex numbers. If $i, j \in \mathbb{Z}$ and $j<i$, then $\sum_{n=i}^{j} a_{n}=0$ and $\prod_{n=i}^{j} a_{n}=1$. The set of natural numbers is given by $\mathbb{N}:=\{1,2,3, \ldots\}$, the set $\mathbb{N}_{0}:=\{0,1,2, \ldots\}=\mathbb{N} \cup\{0\}$, and $\mathbb{Z}:=\{0, \pm 1, \pm 2, \ldots\}$. The sets $\mathbb{Q}$ and $\mathbb{R}$ represents the rational and real numbers respectively. For $d \in \mathbb{N}$, we denote by $\mathbb{R}^{d}$, the finite-dimensional vector space, $d$-dimensional Euclidean space. Furthermore, if $\mathbf{x}, \mathbf{x}^{\prime} \in \mathbb{R}^{d}$ then the Euclidean inner product $(\cdot, \cdot): \mathbb{R}^{d} \times \mathbb{R}^{d} \rightarrow \mathbb{R}$ defined by

$$
\left(\mathbf{x}, \mathbf{x}^{\prime}\right):=x_{1} x_{1}^{\prime}+\cdots+x_{d} x_{d}^{\prime}
$$

induces a norm (the Euclidean norm) $\|\cdot\|: \mathbb{R}^{d} \rightarrow[0, \infty)$, on $\mathbb{R}^{d}$, given by $\|\mathbf{x}\|:=\sqrt{(\mathbf{x}, \mathbf{x})}$.

Please see Appendix A for all notations used in this paper for special functions and orthogonal polynomials.

\section{Fundamental solution of the polyharmonic equation in rotationally-invariant and polyspherical coordinate systems}

In Euclidean space $\mathbb{R}^{d}$, let the Laplacian operator $\Delta: C^{p}\left(\mathbb{R}^{d}\right) \rightarrow C^{p-2}\left(\mathbb{R}^{d}\right)$ for $p \geq 2$ be defined by $\Delta:=\frac{\partial^{2}}{\partial x_{1}^{2}}+\cdots+\frac{\partial^{2}}{\partial x_{d}^{2}}$. If $\Phi: \mathbb{R}^{d} \rightarrow \mathbb{R}$ satisfies the polyharmonic equation given by

$$
(-\Delta)^{k} \Phi(\mathbf{x})=0
$$

where $\mathbf{x} \in \mathbb{R}^{d}, k \in \mathbb{N}$ and $\Phi \in C^{2 k}\left(\mathbb{R}^{d}\right)$, then $\Phi$ is called polyharmonic. We use the nonnegative Laplacian $-\Delta \geq 0$. The inhomogeneous polyharmonic equation is given by

$$
(-\Delta)^{k} \Phi(\mathbf{x})=\rho(\mathbf{x}),
$$

where we take $\rho$ to be an integrable function so that a solution to (2.2) exists. A fundamental solution for the polyharmonic equation on $\mathbb{R}^{d}$ is a function $\mathcal{G}_{k}^{d}:\left(\mathbb{R}^{d} \times \mathbb{R}^{d}\right) \backslash\left\{(\mathbf{x}, \mathbf{x}): \mathbf{x} \in \mathbb{R}^{d}\right\} \rightarrow \mathbb{R}$ which satisfies the equation

$$
(-\Delta)^{k} \mathcal{G}_{k}^{d}\left(\mathbf{x}, \mathbf{x}^{\prime}\right)=\delta\left(\mathbf{x}-\mathbf{x}^{\prime}\right)
$$


where $\delta$ is the Dirac delta function (generalized function/distribution) and $\mathbf{x}^{\prime} \in \mathbb{R}^{d}$. Note that this equation is satisfied in the sense of distributions.

A fundamental solution of the polyharmonic equation is given as follows (see for instance [4], $\left[15\right.$, p. 202], [28, p. 45]). Let $d, k \in \mathbb{N}$. Define $\mathcal{G}_{k}^{d}:\left(\mathbb{R}^{d} \times \mathbb{R}^{d}\right) \backslash\left\{(\mathbf{x}, \mathbf{x}): \mathbf{x} \in \mathbb{R}^{d}\right\} \rightarrow \mathbb{R}$ by

$$
\mathcal{G}_{k}^{d}\left(\mathbf{x}, \mathbf{x}^{\prime}\right):=\left\{\begin{array}{lc}
\frac{(-1)^{k+d / 2+1}\left\|\mathbf{x}-\mathbf{x}^{\prime}\right\|^{2 k-d}}{(k-1) !(k-d / 2) ! 2^{2 k-1} \pi^{d / 2}}\left(\log \left\|\mathbf{x}-\mathbf{x}^{\prime}\right\|-\beta_{k-d / 2, d}\right) \\
\frac{\Gamma(d / 2-k)\left\|\mathbf{x}-\mathbf{x}^{\prime}\right\|^{2 k-d}}{(k-1) ! 2^{2 k} \pi^{d / 2}} & \text { if } d \text { even, } k \geq d / 2,
\end{array}\right.
$$

where $\beta_{p, d} \in \mathbb{Q}$ is defined as $\beta_{p, d}:=\frac{1}{2}\left[H_{p}+H_{d / 2+p-1}-H_{d / 2-1}\right]$, with $H_{j} \in \mathbb{Q}$ being the $j$ th harmonic number $H_{j}:=\sum_{i=1}^{j} \frac{1}{i}$. The gamma function $\Gamma: \mathbb{C} \backslash-\mathbb{N}_{0} \rightarrow \mathbb{C}$, is a natural generalization of the factorial function. Concerning the logarithmic contribution for $d$ even, $k \geq d / 2$, the polynomial $\left\|\mathbf{x}-\mathbf{x}^{\prime}\right\|^{2 k-d}$ is polyharmonic, so any choice for the constant $\beta_{p, d}$ is valid. Our choice for this constant is given such that $-\Delta \mathcal{G}_{k}^{d}=\mathcal{G}_{k-1}^{d}$ is satisfied for all $k>d / 2$, and that for $k=d / 2$, the constant vanishes. Note that a solution of the inhomogeneous polyharmonic equation (2.2) is obtained from $\mathcal{G}_{k}^{d}$ via a convolution.

\subsection{Fundamental solution decompositions in rotationally-invariant coordinate systems}

In this paper we restrict our attention to separable rotationally-invariant coordinate systems for the polyharmonic equation on $\mathbb{R}^{d}$ which are given by

$$
\begin{aligned}
& x_{1}=R\left(\xi_{1}, \ldots, \xi_{d-1}\right) \cos \phi, \\
& x_{2}=R\left(\xi_{1}, \ldots, \xi_{d-1}\right) \sin \phi, \\
& x_{3}=x_{3}\left(\xi_{1}, \ldots, \xi_{d-1}\right), \\
& \ldots \ldots \ldots \ldots \ldots \ldots \ldots . . \\
& x_{d}=x_{d}\left(\xi_{1}, \ldots, \xi_{d-1}\right) .
\end{aligned}
$$

These coordinate systems are described by $d$-coordinates: an angle $\phi \in[0,2 \pi)$ plus $(d-1)$ curvilinear coordinates $\left(\xi_{1}, \ldots, \xi_{d-1}\right)$. Rotationally-invariant coordinate systems parametrize points on the $(d-1)$-dimensional half-hyperplane given by $\phi=$ const and $R \geq 0$ using the curvilinear coordinates $\left(\xi_{1}, \ldots, \xi_{d-1}\right)$. A separable rotationally-invariant coordinate system transforms the polyharmonic equation into a set of $d$-uncoupled ordinary differential equations with separation constants $m \in \mathbb{Z}$ and $k_{j} \in \mathbb{R}$ for $1 \leq j \leq d-2$. For a separable rotationally-invariant coordinate system, this uncoupling is accomplished, in general, by assuming a product solution to $(2.1)$ of the form $\Phi(\mathbf{x})=e^{i m \phi} \mathcal{R}\left(\xi_{1}, \ldots, \xi_{d-1}\right) \prod_{i=1}^{d-1} A_{i}\left(\xi_{i}, m, k_{1}, \ldots, k_{d-2}\right)$, where the properties of the functions $\mathcal{R}$ and $A_{i}$, for $1 \leq i \leq d-1$, and the constants $k_{j}$ for $1 \leq j \leq d-2$, depend on the specific separable rotationally-invariant coordinate system in question. Separable coordinate systems are divided into two different classes, those which are simply separable $(\mathcal{R}=$ const), and those which are $\mathcal{R}$-separable (see [26]).

The Euclidean distance between two points $\mathbf{x}, \mathbf{x}^{\prime} \in \mathbb{R}^{d}$ expressed in the rotationally-invariant coordinate system described in (2.4) is

$$
\left\|\mathbf{x}-\mathbf{x}^{\prime}\right\|=\sqrt{2 R R^{\prime}}\left[\chi-\cos \left(\phi-\phi^{\prime}\right)\right]^{1 / 2}
$$


where the toroidal parameter $\chi$ is

$$
\chi:=\frac{R^{2}+R^{\prime 2}+\sum_{i=3}^{d}\left(x_{i}-x_{i}^{\prime}\right)^{2}}{2 R R^{\prime}} .
$$

The hypersurfaces $\chi=$ const are independent of coordinate system and represent hypertori of revolution.

We now rewrite (2.3) in terms of the rotationally-invariant coordinate system (2.4). From (2.3) we see that, apart from multiplicative constants, the expression $\mathfrak{l}_{k}^{d}:\left(\mathbb{R}^{d} \times \mathbb{R}^{d}\right) \backslash\{(\mathbf{x}, \mathbf{x})$ : $\left.\mathbf{x} \in \mathbb{R}^{d}\right\} \rightarrow \mathbb{R}$ of a fundamental solution for the polyharmonic equation on $\mathbb{R}^{d}$ for $d$ even, $k \geq d / 2$, is given by

$$
\mathfrak{l}_{k}^{d}\left(\mathbf{x}, \mathbf{x}^{\prime}\right):=\left\|\mathbf{x}-\mathbf{x}^{\prime}\right\|^{2 k-d}\left(\log \left\|\mathbf{x}-\mathbf{x}^{\prime}\right\|-\beta_{k-d / 2, d}\right) .
$$

By expressing $\mathfrak{l}_{k}^{d}$ in a rotationally-invariant coordinate system (2.4) we obtain

$$
\begin{aligned}
\mathfrak{l}_{k}^{d}\left(\mathbf{x}, \mathbf{x}^{\prime}\right)= & \left(2 R R^{\prime}\right)^{p}\left[\frac{1}{2} \log \left(2 R R^{\prime}\right)-\beta_{p, d}\right]\left[\chi-\cos \left(\phi-\phi^{\prime}\right)\right]^{p} \\
& +\frac{1}{2}\left(2 R R^{\prime}\right)^{p}\left[\chi-\cos \left(\phi-\phi^{\prime}\right)\right]^{p} \log \left[\chi-\cos \left(\phi-\phi^{\prime}\right)\right],
\end{aligned}
$$

where $p=k-d / 2 \in \mathbb{N}_{0}$. Similarly, when working on an even-dimensional Euclidean space $\mathbb{R}^{d}$ with $1 \leq k \leq d / 2-1$, a fundamental solution of the polyharmonic equation $\mathfrak{h}_{k}^{d}:\left(\mathbb{R}^{d} \times \mathbb{R}^{d}\right) \backslash$ $\left\{(\mathbf{x}, \mathbf{x}): \mathbf{x} \in \mathbb{R}^{d}\right\} \rightarrow(0, \infty)$ is

$$
\mathfrak{h}_{k}^{d}\left(\mathbf{x}, \mathbf{x}^{\prime}\right):=\left\|\mathbf{x}-\mathbf{x}^{\prime}\right\|^{2 k-d} .
$$

By expressing $\mathfrak{h}_{k}^{d}$ in a rotationally-invariant coordinate system we obtain

$$
\mathfrak{h}_{k}^{d}\left(\mathbf{x}, \mathbf{x}^{\prime}\right)=\left(2 R R^{\prime}\right)^{-q}\left[\chi-\cos \left(\phi-\phi^{\prime}\right)\right]^{-q},
$$

where $q=2 k-d \in \mathbb{N}$.

Examining (2.7) and (2.8), we see that for computation of Fourier expansions about the azimuthal separation angle $\left(\phi-\phi^{\prime}\right)$ of $\mathfrak{l}_{k}^{d}$ and $\mathfrak{h}_{k}^{d}$, all that is required is to compute the Fourier cosine series for the following three functions $f_{\chi}, h_{\chi}: \mathbb{R} \rightarrow(0, \infty)$ and $g_{\chi}: \mathbb{R} \rightarrow \mathbb{R}$ defined as

$$
\begin{aligned}
& f_{\chi}(\psi):=(\chi-\cos \psi)^{p}, \quad g_{\chi}(\psi):=(\chi-\cos \psi)^{p} \log (\chi-\cos \psi), \\
& h_{\chi}(\psi):=(\chi-\cos \psi)^{-q},
\end{aligned}
$$

where $p \in \mathbb{N}_{0}, q \in \mathbb{N}$ and $\chi>1$ is a fixed parameter.

The Fourier series of $f_{\chi}$ is given in [8] (cf. (4.4) therein) $)^{1}$, namely for $p \in \mathbb{N}_{0}$,

$$
(z-x)^{p}=\left(z^{2}-1\right)^{p / 2} \sum_{n=0}^{p} \frac{\epsilon_{n}(-p)_{n}(p-n) !}{(p+n) !} P_{p}^{n}\left(\frac{z}{\sqrt{z^{2}-1}}\right) T_{n}(x),
$$

where $\epsilon_{n} \in\{1,2\}$ is the Neumann factor defined by $\epsilon_{n}:=2-\delta_{n, 0}, \delta_{n, 0} \in\{0,1\}$ is the Kronecker delta. The Fourier series of $h_{\chi}$ is given in [8, (4.5)], namely for $p \in \mathbb{N}$,

$$
\frac{1}{(z-x)^{p}}=\frac{\left(z^{2}-1\right)^{-p / 2}}{(p-1) !} \sum_{n=0}^{\infty} \epsilon_{n}(n+p-1) ! P_{p-1}^{-n}\left(\frac{z}{\sqrt{z^{2}-1}}\right) T_{n}(x) .
$$

In order to compute Fourier expansion of $\mathfrak{l}_{k}^{d}(2.7)$ in separable rotationally-invariant coordinate systems, all that remains is to determine the Fourier series of $g_{\chi}$ (see [6]). A discussion of Fourier cosine expansions for a logarithmic fundamental solution of the polyharmonic equation on $\mathbb{R}^{d}$ (from $g_{\chi}$ ) can be found in [7]. The corresponding Gegenbauer polynomial expansions for a logarithmic fundamental solution of the polyharmonic equation on $\mathbb{R}^{d}$ can be found in [6].

\footnotetext{
${ }^{1}$ We have used Whipple's formula (A.10) in (2.9) and (2.10) to convert the associated Legendre functions of the second kind $Q_{\nu}^{\mu}$ appearing in [8] to associated Legendre functions of the first kind $P_{\nu}^{\mu}$.
} 


\section{Jacobi polynomial and limiting expansions for the Euler kernel}

In this section we derive Jacobi, Gegenbauer and Chebyshev polynomial of the first kind series expansions of the Euler kernel $(z-x)^{-\nu}$. These series expansions are used to obtain azimuthal Fourier and hyperspherical harmonic expansions for a fundamental solution of the polyharmonic equation on $\mathbb{R}^{d}$.

Theorem 1. Let $\alpha, \beta>-1$, such that if $\alpha, \beta \in(-1,0)$ then $\alpha+\beta+1 \neq 0, x, z, \nu \in \mathbb{C}$, with $z \in \mathbb{C} \backslash(-\infty, 1]$ on any ellipse with foci at \pm 1 and $x$ in the interior of that ellipse. Then

$$
\begin{aligned}
\frac{1}{(z-x)^{\nu}}= & \frac{(z-1)^{\alpha+1-\nu}(z+1)^{\beta+1-\nu}}{2^{\alpha+\beta+1-\nu}} \\
& \times \sum_{n=0}^{\infty} \frac{(\alpha+\beta+2 n+1) \Gamma(\alpha+\beta+n+1)(\nu)_{n}}{\Gamma(\alpha+1+n) \Gamma(\beta+1+n)} Q_{n+\nu-1}^{(\alpha+1-\nu, \beta+1-\nu)}(z) P_{n}^{(\alpha, \beta)}(x) .
\end{aligned}
$$

Note 1. It has been brought to the author's attention by Tom Koornwinder that Theorem 1 for $\nu=-n, n \in \mathbb{N}_{0}$, specializes to formula (21) in [21], namely

$$
\begin{aligned}
(z-x)^{n}= & (-2)^{n} n ! \Gamma(\alpha+\beta+1) \sum_{k=0}^{n} \frac{(\alpha+\beta+2 k+1)(\alpha+\beta+1)_{k}}{\Gamma(\alpha+\beta+n+k+2)} \\
& \times P_{n-k}^{(-\alpha-n-1,-\beta-n-1)}(z) P_{k}^{(\alpha, \beta)}(x) .
\end{aligned}
$$

This equivalence is provided by the interesting identity

$$
\begin{aligned}
P_{n-k}^{(-\alpha-n-1,-\beta-n-1)}(z)= & \frac{(-1)^{n+k} \Gamma(\alpha+\beta+n+k+2)(z-1)^{\alpha+n+1}(z+1)^{\beta+n+1}}{2^{\alpha+\beta+2 n+1}(n-k) ! \Gamma(\alpha+k+1) \Gamma(\beta+k+1)} \\
& \times Q_{k-n-1}^{(\alpha+n+1, \beta+n+1)}(z),
\end{aligned}
$$

which can be obtained by comparison of Gauss hypergeometric representations.

Proof. Consider the generating function for Gegenbauer polynomials (see, e.g., [27, (18.12.4)])

$$
\frac{1}{\left(1+\rho^{2}-2 \rho x\right)^{\nu}}=\sum_{n=0}^{\infty} \rho^{n} C_{n}^{\nu}(x)=\sum_{n=0}^{\infty} \rho^{n} \frac{(2 \nu)_{n}}{\left(\nu+\frac{1}{2}\right)_{n}} P_{n}^{(\nu-1 / 2, \nu-1 / 2)}(x),
$$

where we have expressed the Gegenbauer polynomial as a symmetric Jacobi polynomial using (A.7). Utilizing (A.6) in (3.2), reversing the order of the summations, and shifting the $n$ index yields

$$
\begin{aligned}
\frac{1}{\left(1+\rho^{2}-2 \rho x\right)^{\nu}}= & \sum_{k=0}^{\infty} \frac{\rho^{k} \Gamma(\alpha+\beta+k+1)}{\Gamma(\alpha+\beta+2 k+1)} P_{k}^{(\alpha, \beta)}(x) \\
& \times \sum_{n=0}^{\infty} \frac{\rho^{n}(2 \nu)_{n+k}\left(\nu+k+\frac{1}{2}\right)_{n}(2 \nu+n+k)_{k}}{n !\left(\nu+\frac{1}{2}\right)_{n+k}} \\
& \times{ }_{3} F_{2}\left(\begin{array}{c}
-n, n+2 \nu+2 k, \alpha+k+1 \\
\nu+k+\frac{1}{2}, \alpha+\beta+2 k+2
\end{array} ; 1\right) .
\end{aligned}
$$

Taking advantage of standard properties such as (A.2), (A.3) produces

$$
\frac{1}{\left(1+\rho^{2}-2 \rho x\right)^{\nu}}=\frac{\sqrt{\pi}}{2^{2 \nu-1} \Gamma(\nu)} \sum_{k=0}^{\infty} \frac{\rho^{k} \Gamma(2 \nu+2 k) \Gamma(\alpha+\beta+k+1)}{\Gamma\left(\nu+k+\frac{1}{2}\right) \Gamma(\alpha+\beta+2 k+1)} P_{k}^{(\alpha, \beta)}(x)
$$




$$
\times \sum_{n=0}^{\infty} \frac{\rho^{n}(2 \nu+2 k)_{n}}{n !}{ }_{3} F_{2}\left(\begin{array}{c}
-n, n+2 \nu+2 k, \alpha+k+1 \\
\nu+k+\frac{1}{2}, \alpha+\beta+2 k+2
\end{array} ; 1\right) .
$$

We substitute the definition of the ${ }_{3} F_{2}$ generalized hypergeometric function (cf. (A.1)) in the sum over $n$, and as previously, reverse the order of the two summations and shift the summation index. It then follows using the duplication formula and (A.2)-(A.5), that one has

$$
\begin{aligned}
& \sum_{n=0}^{\infty} \frac{\rho^{n}(2 \nu+2 k)_{n}}{n !}{ }_{3} F_{2}\left(\begin{array}{c}
-n, n+2 \nu+2 k, \alpha+k+1 \\
\nu+k+\frac{1}{2}, \alpha+\beta+2 k+2
\end{array} ; 1\right) \\
& =\frac{1}{(1-\rho)^{2 \nu+2 k}}{ }_{2} F_{1}\left(\begin{array}{c}
\nu+k, \alpha+k+1 \\
\alpha+\beta+2 k+2
\end{array} ; \frac{-4 \rho}{(1-\rho)^{2}}\right) \text {. }
\end{aligned}
$$

If we apply the right-hand side of (3.3) to (A.13) noting Theorem 12.7.3 (expansion of an analytic function in terms of orthogonal polynomials) in [31] to obtain the regions of convergence, we obtain the desired result.

Corollary 1. Let $\nu \in \mathbb{C} \backslash-\mathbb{N}_{0}$, with $\mu \in\left(-\frac{1}{2}, \infty\right) \backslash\{0\}$, and $z \in \mathbb{C} \backslash(-\infty, 1]$ on any ellipse with foci at \pm 1 with $x$ in the interior of that ellipse. Then

$$
\frac{1}{(z-x)^{\nu}}=\frac{2^{\mu+1 / 2} \Gamma(\mu) e^{i \pi(\mu-\nu+1 / 2)}}{\sqrt{\pi} \Gamma(\nu)\left(z^{2}-1\right)^{(\nu-\mu) / 2-1 / 4}} \sum_{n=0}^{\infty}(n+\mu) Q_{n+\mu-1 / 2}^{\nu-\mu-1 / 2}(z) C_{n}^{\mu}(x) .
$$

Proof. Let $\alpha=\beta=\mu-\frac{1}{2}$ in Theorem 1, and use (A.14) and the definition of the Gegenbauer polynomial in terms of a symmetric Jacobi polynomial (A.7). The points $\nu \in-\mathbb{N}_{0}$ which must be removed are singularities originating from the associated Legendre function of the second kind on the right-hand side of (3.4). This complete the proof.

Note that these singularities are removable and correspond to non-negative integer powers of the binomial $z-x$. See Note 1. These singularities can be removed by taking the limits as $\nu$ approaches them.

Corollary 2. Let $d \geq 3, \nu \in \mathbb{C} \backslash\{0,2,4, \ldots\}, \mathbf{x}, \mathbf{x}^{\prime} \in \mathbb{R}^{d}$ with $r=\|\mathbf{x}\|, r^{\prime}=\left\|\mathbf{x}^{\prime}\right\|$, and $\cos \gamma=\left(\mathbf{x}, \mathbf{x}^{\prime}\right) /\left(r r^{\prime}\right)$. Then

$$
\begin{aligned}
\left\|\mathbf{x}-\mathbf{x}^{\prime}\right\|^{\nu}= & \frac{e^{i \pi(\nu+d-1) / 2} \Gamma\left(\frac{d-2}{2}\right)}{2 \sqrt{\pi} \Gamma\left(-\frac{\nu}{2}\right)} \frac{\left(r_{>}^{2}-r_{<}^{2}\right)^{(\nu+d-1) / 2}}{\left(r r^{\prime}\right)^{(d-1) / 2}} \\
& \times \sum_{n=0}^{\infty}(2 n+d-2) Q_{n+(d-3) / 2}^{(1-\nu-d) / 2}\left(\frac{r^{2}+r^{\prime 2}}{2 r r^{\prime}}\right) C_{n}^{d / 2-1}(\cos \gamma),
\end{aligned}
$$

where $r_{\lessgtr}=\min _{\max }\left\{r, r^{\prime}\right\}$.

Proof. Map $\nu \mapsto-\nu / 2$ in (3.4) and substitute $\left\|\mathbf{x}-\mathbf{x}^{\prime}\right\|=\sqrt{2 r r^{\prime}} \sqrt{z-x}, \mathbf{x}, \mathbf{x}^{\prime} \in \mathbb{R}^{d}$ for $d \geq 3$ (B.3) with $z=\left(r^{2}+r^{\prime 2}\right) /\left(2 r r^{\prime}\right), x=\cos \gamma$, i.e.,

$$
\frac{1}{(z-x)^{\nu}} \mapsto(\sqrt{z-x})^{\nu}=\frac{1}{\left(2 r r^{\prime}\right)^{\nu / 2}}\left\|\mathbf{x}-\mathbf{x}^{\prime}\right\|^{\nu}
$$

This maps the singularities on the right-hand side of (3.4) at $\nu \in-\mathbb{N}_{0}$ to singularities at $\nu=0,2,4, \ldots$. 
Corollary 3. Let $\nu \in \mathbb{C} \backslash-\mathbb{N}_{0}$, and $x, z \in \mathbb{C}$ such that $z \in \mathbb{C} \backslash(-\infty, 1]$ lie on any ellipse with foci at \pm 1 with $x$ in the interior of that ellipse. Then

$$
\frac{1}{(z-x)^{\nu}}=\frac{\sqrt{2} e^{-i \pi(\nu-1 / 2)}}{\sqrt{\pi} \Gamma(\nu)\left(z^{2}-1\right)^{\nu / 2-1 / 4}} \sum_{n=0}^{\infty} \epsilon_{n} T_{n}(x) Q_{n-1 / 2}^{\nu-1 / 2}(z) .
$$

Proof. Take the limit as $\mu \rightarrow 0$ on the right-hand side of (3.4) and use (A.8).

Note that (3.6) is given in $[8,(3.10)]$, so (3.1) and (3.4) represent generalizations of that formula.

Corollary 4. Let $d \geq 2, \nu \in \mathbb{C} \backslash\{0,2,4, \ldots\}, \mathbf{x}, \mathbf{x}^{\prime} \in \mathbb{R}^{d}$. Then

$$
\left\|\mathbf{x}-\mathbf{x}^{\prime}\right\|^{\nu}=\frac{\sqrt{2} e^{i \pi(\nu+1) / 2}\left(2 R R^{\prime}\right)^{\nu / 2}}{\sqrt{2} \Gamma\left(-\frac{\nu}{2}\right)\left(\chi^{2}-1\right)^{-(\nu+1) / 4}} \sum_{m=0}^{\infty} \epsilon_{m} T_{m}\left(\cos \left(\phi-\phi^{\prime}\right)\right) Q_{m-1 / 2}^{-(\nu+1) / 2}(\chi),
$$

where the toroidal parameter $\chi \in[1, \infty)$ is defined in $(2.6)$ such that $\chi=1$ when $\mathbf{x}=\mathbf{x}^{\prime}$.

Proof. Combine (2.5) and Corollary 3.

\section{Addition theorems in Vilenkin's polyspherical coordinates}

In this section we construct power-law addition theorems in Vilenkin's polyspherical coordinates.

\subsection{Power-law addition theorem on $\mathbb{R}^{d}$ for $d \geq 3$ in standard polyspherical coordinates}

In standard polyspherical coordinates (B.11) we have the following multi-summation power-law addition theorem.

Theorem 2. Let $\nu \in \mathbb{C} \backslash\{2 m, 2 m+2,2 m+4, \ldots\}, m \in \mathbb{Z}, \theta_{i} \in[0, \pi]$, for $1 \leq i \leq d-2$, $r, r^{\prime} \in[0, \infty)$ with $d \geq 3$. Then

$$
\begin{aligned}
Q_{m-1 / 2}^{-(\nu+1) / 2}(\chi)= & \sqrt{2} e^{i \pi(d-2) / 2} \pi^{(d-4) / 2}\left(2 r r^{\prime} \prod_{i=1}^{d-2} \sin \theta_{i} \sin \theta_{i}^{\prime}\right)^{-\nu / 2} \\
& \times\left(\chi^{2}-1\right)^{-(\nu+1) / 4} \frac{\left(r_{>}^{2}-r_{<}^{2}\right)(\nu+d-1) / 2}{\left(r r^{\prime}\right)^{(d-1) / 2}} \\
& \times \sum_{l_{d-2}=m}^{\infty} \frac{\left(2 l_{d-2}+1\right)\left(l_{d-2}-m\right) !}{\left(l_{d-2}+m\right) !} \mathrm{P}_{l_{d-2}}^{m}\left(\cos \theta_{d-2}\right) \mathrm{P}_{l_{d-2}}^{m}\left(\cos \theta_{d-2}^{\prime}\right) \\
& \times \sum_{l_{d-3}=l_{d-2}}^{\infty} \Theta_{d-3}^{d}\left(l_{d-3}, l_{d-2} ; \theta_{d-3}\right) \Theta_{d-3}^{d}\left(l_{d-3}, l_{d-2} ; \theta_{d-3}^{\prime}\right) \cdots \\
& \times \sum_{l_{2}=l_{3}}^{\infty} \Theta_{2}^{d}\left(l_{2}, l_{3} ; \theta_{2}\right) \Theta_{2}^{d}\left(l_{2}, l_{3} ; \theta_{2}^{\prime}\right) \\
& \times \sum_{l_{1}=l_{2}}^{\infty} \Theta_{1}^{d}\left(l_{1}, l_{2} ; \theta_{1}\right) \Theta_{1}^{d}\left(l_{1}, l_{2} ; \theta_{1}^{\prime}\right) Q_{l_{1}+(d-3) / 2}^{(1-\nu-d) / 2}\left(\frac{r^{2}+r^{\prime 2}}{2 r r^{\prime}}\right)
\end{aligned}
$$


where

$$
\chi=\frac{r^{2}+r^{\prime 2}-2 r r^{\prime} \sum_{i=1}^{d-2} \cos \theta_{i} \cos \theta_{i}^{\prime} \prod_{j=1}^{i-1} \sin \theta_{j} \sin \theta_{j}^{\prime}}{2 r r^{\prime} \prod_{i=1}^{d-2} \sin \theta_{i} \sin \theta_{i}^{\prime}} .
$$

Proof. If we adopt standard polyspherical coordinates (B.11) (see Fig. 4), then one can obtain an eigenfunction expansion for a power-law fundamental solution of the polyharmonic equation in standard polyspherical coordinates using the Gegenbauer expansion (3.5) with the addition theorem for hyperspherical harmonics (B.13), and the normalized standard hyperspherical harmonics (B.19), obtaining

$$
\begin{aligned}
\left\|\mathbf{x}-\mathbf{x}^{\prime}\right\|^{\nu}= & \frac{4 e^{i \pi(\nu+d-1) / 2} \pi^{(d-1) / 2}}{\Gamma\left(-\frac{\nu}{2}\right)} \frac{\left(r_{>}^{2}-r_{<}^{2}\right)^{(\nu+d-1) / 2}}{\left(r r^{\prime}\right)^{(d-1) / 2}} \\
& \times \sum_{l_{1}, K} Q_{l_{1}+(d-3) / 2}^{(1-\nu-d) / 2}\left(\frac{r^{2}+r^{\prime 2}}{2 r r^{\prime}}\right) Y_{l_{1}}^{K}(\widehat{\mathbf{x}}) \overline{Y_{l_{1}}^{K}\left(\widehat{\mathbf{x}}^{\prime}\right)} .
\end{aligned}
$$

If we expand the product of polyspherical harmonics in (4.2) with (B.19) after reversing the order of the summations, we obtain

$$
\begin{aligned}
\left\|\mathbf{x}-\mathbf{x}^{\prime}\right\|^{\nu}= & \sum_{m=0}^{\infty} \cos \left(m\left(\phi-\phi^{\prime}\right)\right) \frac{\pi^{(d-3) / 2} e^{i \pi(\nu+d-1) / 2} \epsilon_{m}}{\Gamma\left(-\frac{\nu}{2}\right)} \frac{\left(r_{>}^{2}-r_{<}^{2}\right)^{(\nu+d-1) / 2}}{\left(r r^{\prime}\right)^{(d-1) / 2}} \\
& \times \sum_{l_{d-2}=m}^{\infty} \frac{\left(2 l_{d-2}+1\right)\left(l_{d-2}-m\right) !}{\left(l_{d-2}+m\right) !} \mathrm{P}_{l_{d-2}}^{m}\left(\cos \theta_{d-2}\right) \mathrm{P}_{l_{d-2}}^{m}\left(\cos \theta_{d-2}^{\prime}\right) \\
& \times \sum_{l_{d-3}=l_{d-2}}^{\infty} \Theta_{d-3}^{d}\left(l_{d-3}, l_{d-2} ; \theta_{d-3}\right) \Theta_{d-3}^{d}\left(l_{d-3}, l_{d-2} ; \theta_{d-3}^{\prime}\right) \cdots \\
& \times \sum_{l_{2}=l_{3}}^{\infty} \Theta_{2}^{d}\left(l_{2}, l_{3} ; \theta_{2}\right) \Theta_{2}^{d}\left(l_{2}, l_{3} ; \theta_{2}^{\prime}\right) \\
& \times \sum_{l_{1}=l_{2}}^{\infty} \Theta_{1}^{d}\left(l_{1}, l_{2} ; \theta_{1}\right) \Theta_{1}^{d}\left(l_{1}, l_{2} ; \theta_{1}^{\prime}\right) Q_{l_{1}+(d-3) / 2}^{(1-\nu-d) / 2}\left(\frac{r^{2}+r^{\prime 2}}{2 r r^{\prime}}\right),
\end{aligned}
$$

where $\Theta_{j}^{d}\left(l_{j}, l_{j+1} ; \theta\right)$, for $1 \leq j \leq d-2$ is defined in (B.20).

The Fourier expansion for a power-law fundamental solution of the polyharmonic equation in standard polyspherical coordinates is obtained by substituting the expansion in terms of Chebyshev polynomials of the first kind (3.6) in the algebraic expression for a power-law fundamental solution of the polyharmonic equation (cf. (2.5)). This results in

$$
\begin{aligned}
\left\|\mathbf{x}-\mathbf{x}^{\prime}\right\|^{\nu}= & \sqrt{\frac{\pi}{2}} \frac{e^{i \pi(\nu+1) / 2}}{\Gamma(-\nu / 2)}\left(2 r r^{\prime} \prod_{i=1}^{d-2} \sin \theta_{i} \sin \theta_{i}^{\prime}\right)^{\nu / 2}\left(\chi^{2}-1\right)^{(\nu+1) / 4} \\
& \times \sum_{m=-\infty}^{\infty} e^{i m\left(\phi-\phi^{\prime}\right)} Q_{m-1 / 2}^{-(\nu+1) / 2}(\chi) .
\end{aligned}
$$

By comparing the Fourier coefficients of (4.3) with (4.4), we complete the proof of this theorem. 
This is just one example of a derived multi-summation addition theorem for arbitrary dimensions. There are an unlimited number of such straightforward examples to generate. In the next section we derive another example which is valid on $\mathbb{R}^{d}$ where $d$ is given by a power of two, generalized Hopf coordinates (B.12).

\subsection{Power-law addition theorem on $\mathbb{R}^{2^{q}}$ for $q \geq 2$ in generalized Hopf coordinates}

In generalized Hopf coordinates (B.12) we have the following multi-summation power-law addition theorem.

Theorem 3. Let $\nu \in \mathbb{C} \backslash\{2 m, 2 m+2,2 m+4, \ldots\}, m_{1} \in \mathbb{Z}, r, r^{\prime} \in[0, \infty), \vartheta_{i} \in\left[0, \frac{\pi}{2}\right]$ with $1 \leq i \leq 2^{q-1}-1, \phi_{i} \in[0,2 \pi)$ such that $1 \leq i \leq 2^{q-1}$. Then

$$
\begin{aligned}
& Q_{m_{1}-1 / 2}^{-(\nu+1) / 2}(\chi)=\frac{-\left(\prod_{j=1}^{q-1} \cos \vartheta_{2^{j-1}} \cos \vartheta_{2^{j-1}}^{\prime}\right)^{-\nu / 2}}{2^{(\nu+1) / 2}\left(\chi^{2}-1\right)^{(\nu+1) / 4}}\left(\frac{r_{>}^{2}-r_{<}^{2}}{r r^{\prime}}\right)^{\left(\nu+2^{q}-1\right) / 2} \\
& \times \sum_{m_{2}=0}^{\infty} \epsilon_{m_{2}} \cos \left(m_{2}\left(\phi_{2}-\phi_{2}^{\prime}\right)\right) \cdots \sum_{m_{2^{q-1}}=0}^{\infty} \epsilon_{m_{2^{q-1}}} \cos \left(m_{2^{q-1}}\left(\phi_{2^{q-1}}-\phi_{2^{q-1}}^{\prime}\right)\right) \\
& \times \sum_{n_{2^{q-1}-1}=0}^{\infty} \Upsilon_{2^{q-1}-1}^{q}\left(\begin{array}{c}
n_{2^{q-1}-1} \\
\left|m_{2^{q-1}-1}\right|,\left|m_{2^{q-1}}\right|
\end{array} \vartheta_{2^{q-1}-1}\right) \\
& \times \Upsilon_{2^{q-1}-1}^{q}\left(\begin{array}{c}
n_{2^{q-1}-1} \\
\left|m_{2^{q-1}-1}\right|,\left|m_{2^{q-1}}\right|
\end{array} ; \vartheta_{2^{q-1}-1}^{\prime}\right) \cdots \\
& \times \sum_{n_{2^{q-2}}=0}^{\infty} \Upsilon_{2^{q-2}}^{q}\left(\begin{array}{c}
n_{2^{q-2}} \\
\left|m_{1}\right|,\left|m_{2}\right|
\end{array} ; \vartheta_{2^{q-2}}\right) \Upsilon_{2^{q-2}}^{q}\left(\begin{array}{c}
n_{2^{q-2}} \\
\left|m_{1}\right|,\left|m_{2}\right|
\end{array} ; \vartheta_{2^{q-2}}^{\prime}\right)
\end{aligned}
$$

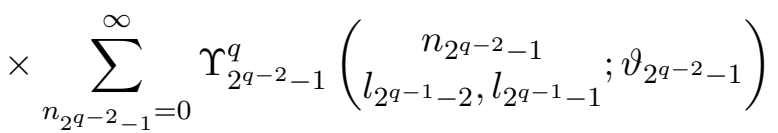

$$
\begin{aligned}
& \times \Upsilon_{2^{q-2}-1}^{q}\left(\begin{array}{c}
n_{2^{q-2}-1} ; \vartheta_{2^{q-2}-1}^{\prime} \\
l_{2^{q-1}-2}, l_{2^{q-1}-1}
\end{array}\right) \cdots \\
& \times \sum_{n_{1}=0}^{\infty} \Upsilon_{1}^{q}\left(\begin{array}{c}
n_{1} \\
l_{2}, l_{3}
\end{array} \vartheta_{1}\right) \Upsilon_{1}^{q}\left(\begin{array}{c}
n_{1} \\
l_{2}, l_{3} ; \vartheta_{1}^{\prime}
\end{array}\right) Q_{2 n_{1}+l_{2}+l_{3}+\left(2^{q}-3\right) / 2}^{\left(1-\nu-2^{q}\right) / 2}\left(\frac{r^{2}+r^{\prime 2}}{2 r r^{\prime}}\right),
\end{aligned}
$$

where

$$
\chi=\frac{r^{2}+{r^{\prime}}^{2}-2 r r^{\prime} \cos \gamma+2 r r^{\prime} \cos \left(\phi_{1}-\phi_{1}^{\prime}\right) \prod_{j=1}^{q-1} \cos \vartheta_{2^{j-1}} \cos \vartheta_{2^{j-1}}^{\prime}}{2 r r^{\prime} \prod_{j=1}^{q-1} \cos \vartheta_{2^{j-1}} \cos \vartheta_{2^{j-1}}^{\prime}} .
$$

Proof. If we adopt generalized Hopf coordinates (B.12) (see Fig. 5), then we can use the corresponding harmonics (B.21) in combination with the addition theorem for hyperspherical harmonics (B.13). We compare the Gegenbauer expansion for powers of the distance (3.5) with the Fourier expansion

$$
\left\|\mathbf{x}-\mathbf{x}^{\prime}\right\|^{\nu}=\frac{\sqrt{2} i e^{i \pi \nu / 2}\left(2 r r^{\prime} \prod_{j=1}^{q-1} \cos \vartheta_{2^{j-1}} \cos \vartheta_{2^{j-1}}^{\prime}\right)^{\nu / 2}}{\sqrt{\pi} \Gamma\left(\frac{-\nu}{2}\right)\left(\chi^{2}-1\right)^{-(\nu+1) / 4}}
$$




$$
\times \sum_{m_{1}=-\infty}^{\infty} e^{i m_{1}\left(\phi_{1}-\phi_{1}^{\prime}\right)} Q_{m_{1}-1 / 2}^{-(\nu+1) / 2}(\chi)
$$

where $\chi>1$ is given by (4.6). Notice that $\chi$ is independent of $\phi_{1}-\phi_{1}^{\prime}$. By using the Gegenbauer expansion (3.5) and inserting the appropriate Gegenbauer polynomial using the addition theorem for hyperspherical harmonics (B.13), we obtain

$$
\begin{aligned}
\left\|\mathbf{x}-\mathbf{x}^{\prime}\right\|^{\nu}= & \frac{-2 i \pi^{2^{q-1}} e^{i \pi \nu / 2}\left(r_{>}^{2}-r_{<}^{2}\right)^{\left(\nu+2^{q}-1\right) / 2}}{\sqrt{\pi} \Gamma\left(\frac{-\nu}{2}\right)\left(r r^{\prime}\right)^{\left(2^{q}-1\right) / 2}} \\
& \times \sum_{l_{1}, K} Q_{l_{1}+\left(2^{q}-3\right) / 2}^{\left(1-\nu-2^{q}\right) / 2}\left(\frac{r^{2}+r^{\prime 2}}{2 r r^{\prime}}\right) Y_{l_{1}}^{K}(\widehat{\mathbf{x}}) \overline{Y_{l_{1}}^{K}\left(\widehat{\mathbf{x}}^{\prime}\right)} .
\end{aligned}
$$

By expanding the product of polyspherical harmonics in (4.8) with (B.21) expressed in terms of surrogate quantum numbers and reversing the order of the sums, we obtain a multi-summation expression for the power of the Euclidean distance between two points in generalized Hopf coordinates. Through comparison of the resulting equation with the $m_{1}$ Fourier coefficients of (4.7), we derive (4.5), a multi-summation addition theorem for the associated Legendre function of the second kind with argument $\chi$ (cf. (4.6)).

\subsection{Power-law addition theorems on $\mathbb{R}^{3}$}

In $d=3$ there are two ways to construct polyspherical coordinates, with trees of type $\mathbf{b}^{\prime} \mathbf{a}$ (see Fig. 2b) and ba (see Fig. 2c). We only treat the first tree since the addition theorem from the second tree is trivially obtained from the first.

\subsubsection{Type ba coordinates}

Corollary 5. Let $\nu \in \mathbb{C} \backslash\{2 m, 2 m+2,2 m+4, \ldots\}, m \in \mathbb{N}_{0}, \theta, \theta^{\prime} \in[0, \pi], r, r^{\prime} \in[0, \infty)$. Then

$$
\begin{aligned}
Q_{m-1 / 2}^{-(\nu+1) / 2}(\chi)= & i \sqrt{\pi} 2^{-(\nu+3) / 2}\left(\sin \theta \sin \theta^{\prime}\right)^{-\nu / 2}\left(\chi^{2}-1\right)^{-(\nu+1) / 4}\left(\frac{r_{>}^{2}-r_{<}^{2}}{r r^{\prime}}\right)^{(\nu+2) / 2} \\
& \times \sum_{l=m}^{\infty}(2 l+1) \frac{(l-m) !}{(l+m) !} Q_{l}^{-(\nu+2) / 2}\left(\frac{r^{2}+r^{\prime 2}}{2 r r^{\prime}}\right) \mathrm{P}_{l}^{m}(\cos \theta) \mathrm{P}_{l}^{m}\left(\cos \theta^{\prime}\right),
\end{aligned}
$$

where

$$
\chi=\frac{r^{2}+{r^{\prime}}^{2}-2 r r^{\prime} \cos \theta \cos \theta^{\prime}}{2 r r^{\prime} \sin \theta \sin \theta^{\prime}} .
$$

Proof. Taking $d=3$ in (4.1) for type ba coordinates converts $\chi$ to (4.10) and the relevant Gegenbauer polynomials reduce to Ferrers functions through (A.12). This completes the proof.

Equation (4.9) is a generalization of one of the main results of [10]. This can be observed if you substitute $\nu=-1$ in (4.9) (this corresponds to a fundamental solution of Laplace's equation on $\mathbb{R}^{3}$ ), then the associated Legendre function of the second kind on the right-hand side reduces to an elementary function through $[1,(8.6 .11)]$, producing

$$
Q_{m-1 / 2}(\chi)=\pi \sqrt{\sin \theta \sin \theta^{\prime}} \sum_{l=|m|}^{\infty} \frac{(l-m) !}{(l+m) !}\left(\frac{r_{<}}{r_{>}}\right)^{l+1 / 2} \mathrm{P}_{l}^{m}(\cos \theta) \mathrm{P}_{l}^{m}\left(\cos \theta^{\prime}\right) .
$$




\subsection{Power-law addition theorems on $\mathbb{R}^{4}$}

In $d=4$ there are five ways to construct polyspherical coordinates, with trees of type $\mathbf{b}^{2} \mathbf{a}$ (see Fig. 3a), bb'a (see Fig. 3b), b'ba (see Fig. 3c), $\mathbf{b}^{\prime 2} \mathbf{a}$ (see Fig. 3d), $\mathbf{c a}^{2}$ (see Fig. 3e). We only treat the first and the fifth trees since the addition theorems from the second, third and fourth trees are trivially obtained from the first.

\subsubsection{Type $\mathrm{b}^{2} \mathrm{a}$ coordinates}

Corollary 6. Let $\nu \in \mathbb{C} \backslash\{2 m, 2 m+2,2 m+4, \ldots\}, m \in \mathbb{Z}, r, r^{\prime} \in[0, \infty), \theta_{1}, \theta_{1}^{\prime}, \theta_{2}, \theta_{2}^{\prime} \in[0, \pi]$. Then

$$
\begin{aligned}
Q_{m-1 / 2}^{-(\nu+1) / 2}(\chi)= & \frac{-1}{2^{(\nu+1) / 2}}\left(\frac{r_{>}^{2}-r_{<}^{2}}{r r^{\prime}}\right)^{(\nu+3) / 2}\left(\chi^{2}-1\right)^{-(\nu+1) / 4}\left(\sin \theta_{1} \sin \theta_{1}^{\prime} \sin \theta_{2} \sin \theta_{2}^{\prime}\right)^{-\nu / 2} \\
& \times \sum_{l_{2}=|m|}^{\infty} \frac{2^{2 l_{2}}\left(2 l_{2}+1\right)\left(l_{2} !\right)^{2}\left(l_{2}-m\right) !}{\left(l_{2}+m\right) !}\left(\sin \theta_{1} \sin \theta_{1}^{\prime}\right)^{l_{2}} \mathrm{P}_{l_{2}}^{m}\left(\cos \theta_{2}\right) \mathrm{P}_{l_{2}}^{m}\left(\cos \theta_{2}^{\prime}\right) \\
& \times \sum_{l_{1}=l_{2}}^{\infty} \frac{\left(l_{1}+1\right)\left(l_{1}-l_{2}\right) !}{\left(l_{1}+l_{2}+1\right) !} Q_{l_{1}+1 / 2}^{-(\nu+3) / 2}\left(\frac{r^{2}+r^{\prime 2}}{2 r r^{\prime}}\right) \\
& \times C_{l_{1}-l_{2}}^{l_{2}+1}\left(\cos \theta_{1}\right) C_{l_{1}-l_{2}}^{l_{2}+1}\left(\cos \theta_{1}^{\prime}\right),
\end{aligned}
$$

where

$$
\chi=\frac{r^{2}+r^{\prime 2}-2 r r^{\prime} \cos \theta_{1} \cos \theta_{1}^{\prime}-2 r r^{\prime} \sin \theta_{1} \sin \theta_{1}^{\prime} \cos \theta_{2} \cos \theta_{2}^{\prime}}{2 r r^{\prime} \sin \theta_{1} \sin \theta_{1}^{\prime} \sin \theta_{2} \sin \theta_{2}^{\prime}} .
$$

Proof. Taking $d=4$ in (4.1) completes the proof.

If you substitute $\nu=-2$ (a fundamental solution for the Laplacian on $\mathbb{R}^{4}$ ) in (4.11) then the Legendre functions of the second kind reduce to elementary functions through $[1,(8.6 .10-11)]$, and one obtains the following

$$
\begin{aligned}
\frac{\left(\chi^{2}-1\right)^{-1 / 2}}{\left(\chi+\sqrt{\chi^{2}-1}\right)^{m}}= & 2 \sin \theta_{1} \sin \theta_{1}^{\prime} \sin \theta_{2} \sin \theta_{2}^{\prime} \\
& \times \sum_{l_{2}=|m|}^{\infty} \frac{2^{2 l_{2}}\left(2 l_{2}+1\right)\left(l_{2} !\right)^{2}\left(l_{2}-m\right) !}{\left(l_{2}+m\right) !}\left(\sin \theta_{1} \sin \theta_{1}^{\prime}\right)^{l_{2}} \mathrm{P}_{l_{2}}^{m}\left(\cos \theta_{2}\right) \mathrm{P}_{l_{2}}^{m}\left(\cos \theta_{2}^{\prime}\right) \\
& \times \sum_{l_{1}=l_{2}}^{\infty} \frac{\left(l_{1}-l_{2}\right) !}{\left(l_{1}+l_{2}+1\right) !}\left(\frac{r_{<}}{r_{>}}\right)^{l_{1}+1} C_{l_{1}-l_{2}}^{l_{2}+1}\left(\cos \theta_{1}\right) C_{l_{1}-l_{2}}^{l_{2}+1}\left(\cos \theta_{1}^{\prime}\right) .
\end{aligned}
$$

\subsubsection{Type $\mathrm{ca}^{2}$ coordinates}

Corollary 7. Let $\nu \in \mathbb{C} \backslash\{2 m, 2 m+2,2 m+4, \ldots\}, m_{1} \in \mathbb{Z}, r, r^{\prime} \in[0, \infty), \vartheta, \vartheta^{\prime} \in\left[0, \frac{\pi}{2}\right]$, $\phi_{2}, \phi_{2}^{\prime} \in[0,2 \pi)$. Then

$$
\begin{aligned}
Q_{m_{1}-1 / 2}^{-(\nu+1) / 2}(\chi)= & \frac{-2^{-(\nu+1) / 2}}{\left(\chi^{2}-1\right)^{(\nu+1) / 4}}\left(\frac{r_{>}^{2}-r_{<}^{2}}{r r^{\prime}}\right)^{(\nu+3) / 2}\left(\cos \vartheta \cos \vartheta^{\prime}\right)^{\left|m_{1}\right|-\nu / 2} \\
& \times \sum_{m_{2}=0}^{\infty} \epsilon_{m_{2}} \cos \left(m_{2}\left(\phi_{2}-\phi_{2}^{\prime}\right)\right)\left(\sin \vartheta \sin \vartheta^{\prime}\right)^{m_{2}}
\end{aligned}
$$




$$
\begin{aligned}
& \times \sum_{n=0}^{\infty} \frac{\left(2 n+\left|m_{1}\right|+m_{2}+1\right)\left(\left|m_{1}\right|+m_{2}+n\right) ! n !}{\left(\left|m_{1}\right|+n\right) !\left(m_{2}+n\right) !} \\
& \times Q_{2 n+\left|m_{1}\right|+m_{2}+1 / 2}^{-(\nu+3) / 2}\left(\frac{r^{2}+r^{\prime 2}}{2 r r^{\prime}}\right) P_{n}^{\left(m_{2},\left|m_{1}\right|\right)}(\cos 2 \vartheta) P_{n}^{\left(m_{2},\left|m_{1}\right|\right)}\left(\cos 2 \vartheta^{\prime}\right),
\end{aligned}
$$

where

$$
\chi=\frac{r^{2}+r^{\prime 2}-2 r r^{\prime} \sin \vartheta \sin \vartheta^{\prime} \cos \left(\phi_{2}-\phi_{2}^{\prime}\right)}{2 r r^{\prime} \cos \vartheta \cos \vartheta^{\prime}} .
$$

Proof. Taking $q=2$ in (4.5) completes the proof.

If you substitute $\nu=-2$ in (4.12), then the Legendre functions of the second kind reduce to elementary functions through $[1,(8.6 .10-11)]$, and one obtains the following

$$
\begin{aligned}
\frac{\left(\chi^{2}-1\right)^{-1 / 2}}{\left(\chi+\sqrt{\chi^{2}-1}\right)^{m_{1}}}= & 2\left(\cos \vartheta \cos \vartheta^{\prime}\right)^{\left|m_{1}\right|+1} \sum_{m_{2}=0}^{\infty} \epsilon_{m_{2}} \cos \left[m_{2}\left(\phi_{2}-\phi_{2}^{\prime}\right)\right]\left(\sin \vartheta \sin \vartheta^{\prime}\right)^{\left|m_{2}\right|} \\
& \times \sum_{n=0}^{\infty} \frac{\left(\left|m_{1}\right|+\left|m_{2}\right|+n\right) ! n !}{\left(\left|m_{1}\right|+n\right) !\left(\left|m_{2}\right|+n\right) !}\left(\frac{r_{<}}{r_{>}}\right)^{\left|m_{1}\right|+\left|m_{2}\right|+2 n+1} \\
& \times P_{n}^{\left(\left|m_{2}\right|,\left|m_{1}\right|\right)}(\cos 2 \vartheta) P_{n}^{\left(\left|m_{2}\right|,\left|m_{1}\right|\right)}\left(\cos 2 \vartheta^{\prime}\right) .
\end{aligned}
$$

Note that in the addition theorems (4.12) and (4.13), that if you make the map $\vartheta \mapsto \vartheta-\frac{\pi}{2}$, then this transformation preserves the addition theorems such that $m_{1} \leftrightarrow m_{2}$. This transformation is equivalent to swapping the position of $\phi_{1}$ and $\phi_{2}$ for the tree in Fig. 3e.

\section{A Special functions and orthogonal polynomials}

The generalized hypergeometric function ${ }_{p} F_{q}: \mathbb{C}^{p} \times\left(\mathbb{C} \backslash-\mathbb{N}_{0}\right)^{q} \times\{z \in \mathbb{C}:|z|<1\} \rightarrow \mathbb{C}$ can be defined as

$$
{ }_{p} F_{q}\left(\begin{array}{l}
a_{1}, \ldots, a_{p} \\
b_{1}, \ldots, b_{q}
\end{array} ; z\right):=\sum_{n=0}^{\infty} \frac{\left(a_{1}\right)_{n} \cdots\left(a_{p}\right)_{n}}{\left(b_{1}\right)_{n} \cdots\left(b_{q}\right)_{n}} \frac{z^{n}}{n !},
$$

where the Pochhammer symbol (rising factorial) $(\cdot)_{n}: \mathbb{C} \rightarrow \mathbb{C}$ for $n \in \mathbb{N}_{0}$ is defined by $(z)_{n}:=$ $\prod_{i=1}^{n}(z+i-1)$. Furthermore one has

$$
(z)_{n}=\frac{\Gamma(z+n)}{\Gamma(z)}
$$

for $z \in \mathbb{C} \backslash-\mathbb{N}_{0}$, which implies

$$
(z)_{n+k}=(z)_{k}(z+k)_{n},
$$

$k \in \mathbb{N}_{0}, z \in \mathbb{C}$. One also has

$$
(-n-k)_{k}=\frac{(-1)^{k}(n+k) !}{n !} .
$$

In this paper, we will use two different generalized hypergeometric functions, namely ${ }_{3} F_{2}$ and the Gauss hypergeometric function ${ }_{2} F_{1}$ (see for instance Chapter 15 in [27]). We also use the binomial expansions for $p=1, q=0$, namely

$$
{ }_{1} F_{0}\left(\begin{array}{l}
\alpha \\
-
\end{array} z\right)=(1-z)^{-\alpha}
$$


where $\alpha, z \in \mathbb{C}$ such that $|z|<1$. The special functions used in this paper as well as and their properties can be described in terms of these.

There are many important orthogonal polynomials which can be defined in terms of a terminating generalized hypergeometric series. The Jacobi polynomials $P_{n}^{(\alpha, \beta)}: \mathbb{C} \rightarrow \mathbb{C}$, for $n \in \mathbb{N}_{0}$, and $\alpha, \beta>-1$ such that if $\alpha, \beta \in(-1,0)$ then $\alpha+\beta+1 \neq 0$, are defined as [27, (18.5.7)]

$$
P_{n}^{(\alpha, \beta)}(z):=\frac{(\alpha+1)_{n}}{n !}{ }_{2} F_{1}\left(\begin{array}{c}
-n, n+\alpha+\beta+1 \\
\alpha+1
\end{array} ; \frac{1-z}{2}\right) .
$$

These polynomials are orthogonal with respect to the positive weight $w:(-1,1) \rightarrow[0, \infty)$, $w(x):=(1-x)^{\alpha}(1+x)^{\beta}$, with orthogonality relation

$$
\int_{-1}^{1} P_{m}^{(\alpha, \beta)}(x) P_{n}^{(\alpha, \beta)}(x)(1-x)^{\alpha}(1+x)^{\beta} d x=\frac{\delta_{m, n}}{\left(N_{n}^{\alpha, \beta}\right)^{2}} .
$$

The normalization constant $N_{n}^{\alpha, \beta} \in(0, \infty)$ is given by

$$
N_{n}^{\alpha, \beta}=\sqrt{\frac{(2 n+\alpha+\beta+1) \Gamma(n+\alpha+\beta+1) n !}{2^{\alpha+\beta+1} \Gamma(n+\alpha+1) \Gamma(n+\beta+1)}} .
$$

The connection relation for Jacobi polynomials with two free parameters is given by (see for instance [17, p. 256])

$$
P_{n}^{(\gamma, \delta)}(x)=\sum_{k=0}^{n} c_{n, k}(\gamma, \delta ; \alpha, \beta) P_{k}^{(\alpha, \beta)}(x),
$$

where $\gamma, \delta>-1$, and such that if $\gamma, \delta \in(-1,0)$ then $\gamma+\delta+1 \neq 0$,

$$
\begin{aligned}
c_{n, k}(\gamma, \delta ; \alpha, \beta)= & \frac{(\gamma+k+1)_{n-k}(n+\gamma+\delta+1)_{k} \Gamma(\alpha+\beta+k+1)}{(n-k) ! \Gamma(\alpha+\beta+2 k+1)} \\
& \times{ }_{3} F_{2}\left(\begin{array}{c}
-n+k, n+k+\gamma+\delta+1, \alpha+k+1 \\
\gamma+k+1, \alpha+\beta+2 k+2
\end{array} ;\right) .
\end{aligned}
$$

Jacobi polynomials with parameters $\alpha=\beta$ are described as symmetric and are representable in terms of Gegenbauer polynomials using (see (6.4.9) in [3])

$$
C_{n}^{\nu}(x)=\frac{(2 \nu)_{n}}{\left(\nu+\frac{1}{2}\right)_{n}} P_{n}^{(\nu-1 / 2, \nu-1 / 2)}(x),
$$

where $\nu \in\left(-\frac{1}{2}, \infty\right) \backslash\{0\}$. The Chebyshev polynomial of the first kind $T_{n}: \mathbb{C} \rightarrow \mathbb{C}$ is defined as (see $\S 5.7 .2$ in $[25])$

$$
T_{n}(z):={ }_{2} F_{1}\left(\begin{array}{c}
-n, n \\
\frac{1}{2}
\end{array} ; \frac{1-z}{2}\right)
$$

for $n \in \mathbb{N}_{0}$. These can be computed in terms of Gegenbauer polynomials using

$$
\lim _{\mu \rightarrow 0} \frac{n+\mu}{\mu} C_{n}^{\mu}(x)=\epsilon_{n} T_{n}(x)
$$

(see for instance (6.4.13) in [3]). 
The associated Legendre function of the second kind $Q_{\nu}^{\mu}: \mathbb{C} \backslash(-\infty, 1] \rightarrow \mathbb{C}, \nu+\mu \notin-\mathbb{N}$, can be defined in terms of the Gauss hypergeometric function as follows [27, (14.3.7) and $\S 14.21]$

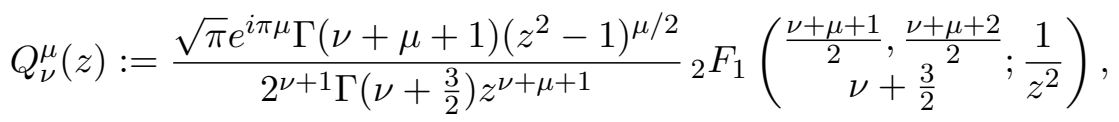

for $|z|>1$ and elsewhere in $z$ by analytic continuation of the Gauss hypergeometric function. One may also define the associated Legendre function of the second kind using [25, entry 24, p. 161]

$$
Q_{\nu}^{\mu}(z):=\frac{e^{i \pi \mu} 2^{\nu} \Gamma(\nu+1) \Gamma(\nu+\mu+1)(z+1)^{\mu / 2}}{\Gamma(2 \nu+2)(z-1)^{b / 2+a+1}}{ }_{2} F_{1}\left(\begin{array}{c}
\nu+1, \nu+\mu+1 \\
2+2 \nu
\end{array} \frac{2}{1-z}\right),
$$

for $|1-z|>2$. Similarly, the associated Legendre function of the first kind can be defined using the Gauss hypergeometric function [27, (14.3.6) and $\S 14.21(\mathrm{i})]$

$$
P_{\nu}^{\mu}(z):=\frac{1}{\Gamma(1-\mu)}\left(\frac{z+1}{z-1}\right)^{\mu / 2} F_{1}\left(\begin{array}{c}
-\nu, \nu+1 \\
1-\mu
\end{array} ; \frac{1-z}{2}\right)
$$

where $|1-z|<2$, and elsewhere in $z$ by analytic continuation. We can use Whipple's formula to relate the associated Legendre function of the first kind with the associated Legendre function of the second kind. It is given by [1, (8.2.7)]

$$
P_{-\mu-1 / 2}^{-\nu-1 / 2}\left(\frac{z}{\sqrt{z^{2}-1}}\right)=\sqrt{\frac{2}{\pi}} \frac{\left(z^{2}-1\right)^{1 / 4} e^{-i \mu \pi}}{\Gamma(\nu+\mu+1)} Q_{\nu}^{\mu}(z),
$$

for $\operatorname{Re} z>0$. The Ferrers function of the first kind (associated Legendre function of the first kind on-the-cut) $\mathrm{P}_{\nu}^{\mu}:(-1,1) \rightarrow \mathbb{C}$ can be defined as $[27,(14.3 .1)]$

$$
\mathrm{P}_{\nu}^{\mu}(x):=\frac{1}{\Gamma(1-\mu)}\left(\frac{1+x}{1-x}\right)^{\mu / 2}{ }_{2} F_{1}\left(\begin{array}{c}
-\nu, \nu+1 \\
1-\mu
\end{array} ; \frac{1-x}{2}\right) .
$$

There is a relation between certain Gegenbauer polynomials on $(-1,1)$ and the Ferrers function of the first kind (cf. (8.936.2) in [16]), namely

$$
C_{l-m}^{m+1 / 2}(x)=\frac{(-1)^{m}\left(1-x^{2}\right)^{-m / 2}}{(2 m-1) ! !} \mathrm{P}_{l}^{m}(x),
$$

where the double factorial $:\{-1,0,1, \ldots\} \rightarrow \mathbb{N}$ is defined such that

$$
n ! !:= \begin{cases}n \cdot(n-2) \cdots 2 & \text { if } n \text { even } \geq 2 \\ n \cdot(n-2) \cdots 1 & \text { if } n \text { odd } \geq 1 \\ 1 & \text { if } n=-1,0\end{cases}
$$

The Jacobi function of the second kind $Q_{\gamma}^{(\alpha, \beta)}: \mathbb{C} \backslash(-\infty, 1] \rightarrow \mathbb{C}$ (cf. [13, (10.8.18)]) is defined by

$$
Q_{\gamma}^{(\alpha, \beta)}(z):=\frac{2^{\alpha+\beta+\gamma} \Gamma(\alpha+\gamma+1) \Gamma(\beta+\gamma+1)}{\Gamma(\alpha+\beta+2 \gamma+2)(z-1)^{\alpha+\gamma+1}(z+1)^{\beta}}{ }_{2} F_{1}\left(\begin{array}{c}
\gamma+1, \alpha+\gamma+1 \\
\alpha+\beta+2 \gamma+2
\end{array} ; \frac{2}{1-z}\right),
$$

where $\alpha+\gamma, \beta+\gamma \notin-\mathbb{N}$. We can derive a relation between the symmetric Jacobi function of the second kind and the associated Legendre function of the second kind

$$
Q_{n+\nu-1}^{(\mu-\nu+1 / 2, \mu-\nu+1 / 2)}(z)=\frac{2^{\mu-\nu+1 / 2} \Gamma(\mu+n+1 / 2) e^{i \pi(\mu-\nu+1 / 2)}}{\Gamma(\nu+n)\left(z^{2}-1\right)^{(\mu-\nu) / 2+1 / 4}} Q_{n+\mu-1 / 2}^{\nu-\mu-1 / 2}(z),
$$

where $n \in \mathbb{N}_{0}, \mu \in \mathbb{C} \backslash\left\{-\frac{1}{2},-\frac{3}{2},-\frac{5}{2}, \ldots\right\}, \nu \in \mathbb{C} \backslash-\mathbb{N}_{0}$. The relation (A.14) can be verified by comparing (A.13) with (A.9). 

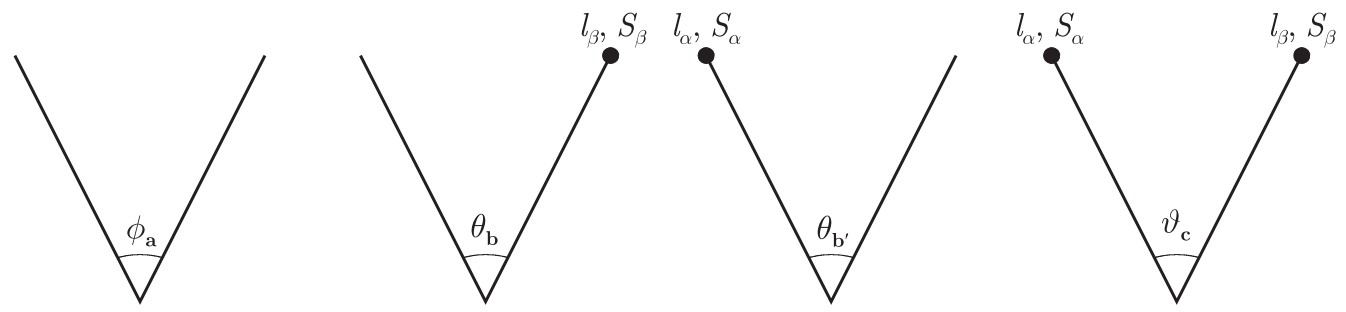

Figure 1. This figure shows the possibilities from left to right for branching nodes of type $\mathbf{a}, \mathbf{b}, \mathbf{b}^{\prime}$ and $\mathbf{c}$. For type $\mathbf{a}$, both branches end on a leaf node. The angle corresponding to this type of branching node is $\phi_{\mathbf{a}} \in[0,2 \pi)$. For type $\mathbf{b}$, the left branch ends on a leaf node and the right branch ends on a branching node. The angle corresponding to this type of branching node is $\theta_{\mathbf{b}} \in[0, \pi]$. For type $\mathbf{b}^{\prime}$, the left-branch ends on a branching node and the right branch ends on a leaf node. The angle corresponding to this type of branching node is $\theta_{\mathbf{b}^{\prime}} \in\left[-\frac{\pi}{2}, \frac{\pi}{2}\right]$. For type $\mathbf{c}$, both the left and right branches ends on branching nodes (branching nodes of type $\mathbf{c}$ are only possible for $d \geq 4$ ). The angle corresponding to this type of branching node is $\vartheta_{\mathbf{c}} \in\left[0, \frac{\pi}{2}\right]$.

\section{B Vilenkin's polyspherical coordinates and the method of trees}

Polyspherical coordinates are hyperspherical coordinates which are described by a radial coordinate $r \in[0, \infty)$ plus $(d-1)$-angles which together parametrize points on $\mathbb{S}_{r}^{d-1}{ }^{2}$ We will first discuss a general procedure for constructing polyspherical coordinate systems called the "method of trees" 3 . We will give some examples of polyspherical coordinates which will be used in the rest of the paper and then describe the harmonic separated product solutions.

In these rooted trees, there are two types of nodes, leaf nodes and branching nodes. For a coordinate system on $\mathbb{R}^{d}$, there are $d$-leaf nodes, each corresponding to the particular Cartesian component of an arbitrary position vector $\mathbf{x} \in \mathbb{R}^{d}$. The branching nodes split into two separate branches, one up to the left and one up to the right. Each branch emanating from a branching node will end on either a leaf node or on another branching node. There are four possibilities for branching nodes (see Fig. 1).

Separation of variables in polyspherical coordinates with $(d-1)$-angles, for Laplace's equation on $\mathbb{R}^{d}$ produces $(d-1)$-separation constants, each of which are called quantum numbers. The quantum numbers corresponding to these angles are all integers. Quantum numbers for a particular tree label the basis of separable solutions for Laplace's equation in that particular coordinate system. With each branching node of the tree, we associate a quantum number as well as an angle. The quantum number corresponding to a $(2 \pi)$-periodic (azimuthal) angle is called an azimuthal quantum number. Each azimuthal angle corresponds to a branching node of type a and an azimuthal quantum number $m \in \mathbb{Z}$. A natural consequence of the method of trees is that there must exist at least one azimuthal angle for each tree, and therefore also for each polyspherical coordinate system.

Branching nodes of type $\mathbf{b}, \mathbf{b}^{\prime}$ and $\mathbf{c}$ (see Fig. 1), are associated with angles, which in turn are associated with quantum numbers which we refer to as angular momentum quantum numbers $l \in \mathbb{N}_{0}$ (see for instance Chapter 10 in [14]). There is always at least one branching node, the root node, and all branching nodes correspond to a particular angle and quantum number. Let us associate each branching node with an angle and its corresponding quantum number. These trees

\footnotetext{
${ }^{2}$ The Riemannian manifold $\mathbb{S}_{r}^{d}$ is defined as the set of all points in $\mathbb{R}^{d+1}$ such that $x_{0}^{2}+\cdots+x_{d}^{2}=r^{2}(r>0)$, with the metric induced from that of the ambient Euclidean space. We denote the $d$-dimensional hypersphere of unit radius as $\mathbb{S}^{d}:=\mathbb{S}_{1}^{d}$.

${ }^{3}$ Describing polyspherical coordinate systems in terms of rooted trees was originally developed in [34] (see also $[32, \S 9.5],[33, \S 10.5])$ and has since been used extensively by others in a variety of contexts (see for instance $[18,19,20])$.
} 
parametrize points in polyspherical coordinates as follows. Starting at the root node, traverse the tree upward until you reach the leaf node corresponding to $x_{i}$. The parametrization for $x_{i}$ is given by the hyperspherical radius $r$ multiplied by cosine or sine of each angle encountered as you traverse the tree upward until you reach the leaf node corresponding to $x_{i}$. If you branch upwards to the left or upwards to the right at each branching node, multiply by the cosine or sine of the corresponding angle respectively. This procedure produces the appropriate transformation from polyspherical coordinates to Cartesian coordinates.

There are large numbers of equivalent trees and an even larger number of total trees, each with their own specific polyspherical coordinate system. The enumeration of these trees are characterized as follows. For $d, b_{d} \in \mathbb{N}$, let $b_{d}$ be the total number of trees. Then $b_{1}=1$ is the number of possible 1-branch trees. In our context, a 1-branch tree does not exist in isolation. The following recurrence relation gives the total number of trees for arbitrary dimension

$$
b_{d}=\sum_{i=1}^{d-1} b_{i} b_{d-i}
$$

Using the recurrence relation (B.1), the first few elements of the sequence are given by

$$
\left(b_{d}: d \in\{2, \ldots, 13\}\right)=(1,2,5,14,42,132,429,1430,4862,16796,58786,208012) .
$$

The total number of trees are given in terms of the Catalan numbers $C_{n}$ (see for instance Sloane integer sequence A000108 [29] or p. 200 in [30]), i.e., $b_{d}=C_{d-1}=\frac{1}{d}\left(\begin{array}{c}2 d-2 \\ d-1\end{array}\right)$, where $\left(\begin{array}{l}n \\ k\end{array}\right)=\frac{n !}{k !(n-k) !}$ is the binomial coefficient for $k, n \in \mathbb{N}_{0}$ with $0 \leq k \leq n$. If $a_{d} \in \mathbb{N}$ is the total number of equivalence classes for equivalent trees for a given dimension (determined by a left-right symmetry in the topology of the trees), then $a_{d}$ satisfies the following recurrence relation

$$
a_{d}= \begin{cases}\sum_{i=1}^{\lfloor d / 2\rfloor} a_{i} a_{d-i}, & \text { if } d \text { odd } \\ \sum_{i=1}^{d / 2-1} a_{i} a_{d-i}+\frac{1}{2} a_{d / 2}\left(a_{d / 2}+1\right), & \text { if } d \text { even. }\end{cases}
$$

Using the recurrence relation (B.2), the first few elements of the sequence are given by

$$
\left(a_{d}: d \in\{2, \ldots, 13\}\right)=(1,1,2,3,6,11,23,46,98,207,451,983) .
$$

These are given in terms of the Wedderburn-Etherington numbers (see for instance Sloane integer sequence A001190 [29]). We use a left-to-right recursive naming language for our trees based on a depth-first search (see [12, pp. 540-549]). This naming language is given by listing the types of branching nodes available in a particular tree.

In a polyspherical coordinate system the Euclidean distance between two points (2.5) can also be given as

$$
\left\|\mathbf{x}-\mathbf{x}^{\prime}\right\|=\sqrt{2 r r^{\prime}}\left[\frac{r^{2}+r^{\prime 2}}{2 r r^{\prime}}-\cos \gamma\right]^{1 / 2},
$$

where the separation angle $\gamma \in[0, \pi]$ is defined through the relation

$$
\cos \gamma:=\frac{\left(\mathbf{x}, \mathbf{x}^{\prime}\right)}{\|\mathbf{x}\|\left\|\mathbf{x}^{\prime}\right\|}
$$

using the Euclidean inner product and norm (cf. (1.1)). The method of trees constructs the cosine of the separation angle (B.4) in a direct manner. The cosine of the separation angle will 


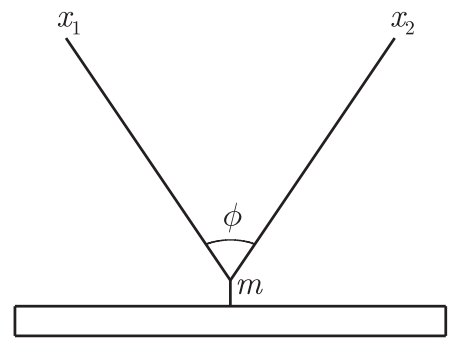

(a)

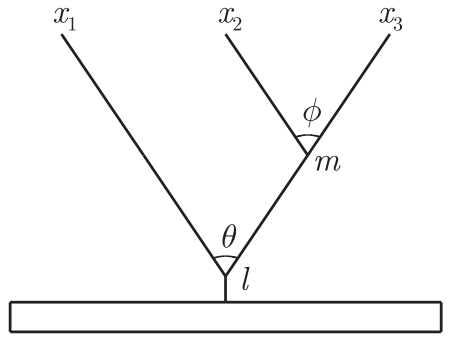

(b)

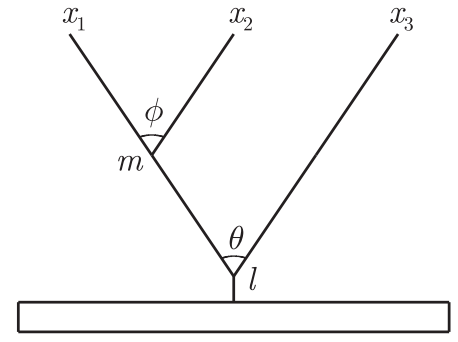

(c)

Figure 2. Tree diagrams for two and three dimensional polyspherical coordinate systems of type: (a) a, (b) ba, (c) $\mathbf{b}^{\prime} \mathbf{a}$.

be given by the sum of $d$-terms, each corresponding to a leaf node of the tree. There is a unique path starting from the root node to each leaf node. It is

$$
\cos \gamma=\sum_{i=1}^{d} \prod_{j=1}^{N_{i}} A_{i, j}\left(\psi_{i, j}\right) A_{i, j}\left(\psi_{i, j}^{\prime}\right),
$$

where $N_{i}$ is the number of branching nodes encountered from the root node to the leaf node, $A_{i, j}: \mathbb{R} \rightarrow[-1,1]$ is either the trigonometric cosine or sine function depending respectively on whether the left branch or right branch is chosen respectively, and $\psi_{i, j} \in \mathbb{R}$ is the angle corresponding to the $j$ th branching node for each $i$ th leaf node. The formula for the cosine of the separation angle is unique for each tree.

\section{B.1 Examples of Vilenkin's polyspherical coordinate systems}

The simplest example of a polyspherical coordinate system on $\mathbb{R}^{d}$ occurs for $d=2$ (polar coordinates) where there is one branching node (the root node) and two leaf nodes (see Figs. 2a and 5a). The left-branch ends on the leaf node corresponding to $x_{1}$ and the right branch ends on the leaf node corresponding to $x_{2}$, i.e.,

$$
x_{1}=r \cos \phi, \quad x_{2}=r \sin \phi,
$$

where $r \in[0, \infty)$ and $\phi \in[0,2 \pi)$. We refer to this tree as type a. The cosine of the separation angle is given by

$$
\cos \gamma=\cos \left(\phi-\phi^{\prime}\right)
$$

and corresponding to the angle $\phi$ is the azimuthal quantum number $m \in \mathbb{Z}$ (see Fig. 2a).

In $d=3$ there are two possible topological trees, each corresponding to one of two different trees. The first tree (see Fig. 2b) corresponds to the coordinate system

$$
x_{1}=r \cos \theta, \quad x_{2}=r \sin \theta \cos \phi, \quad x_{3}=r \sin \theta \sin \phi,
$$

where $\theta \in[0, \pi], \phi \in[0,2 \pi)$, i.e., standard spherical coordinates. This is a tree of type ba. The cosine of the separation angle is given by

$$
\cos \gamma=\cos \theta \cos \theta^{\prime}+\sin \theta \sin \theta^{\prime} \cos \left(\phi-\phi^{\prime}\right),
$$

and corresponding to the angles $\theta \in[0, \pi]$ and $\phi \in[0,2 \pi)$ are the quantum numbers $l \in \mathbb{N}_{0}$ and $m \in \mathbb{Z}$ respectively. The second tree (see Fig. 2c) is of type $\mathbf{b}^{\prime} \mathbf{a}$ and is equivalent to the first. 


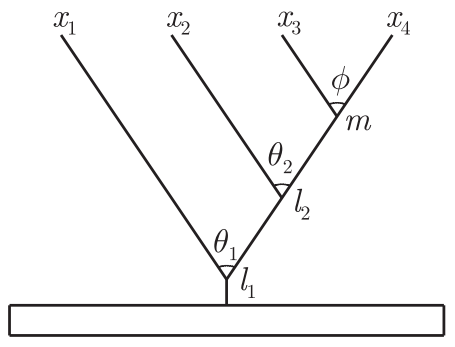

(a)

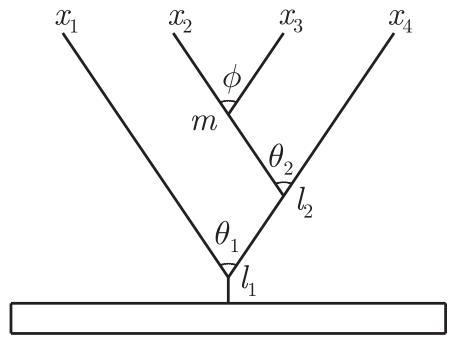

(b)

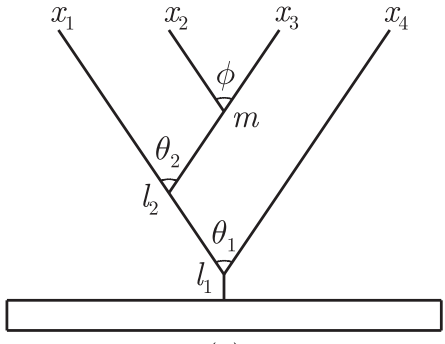

(c)

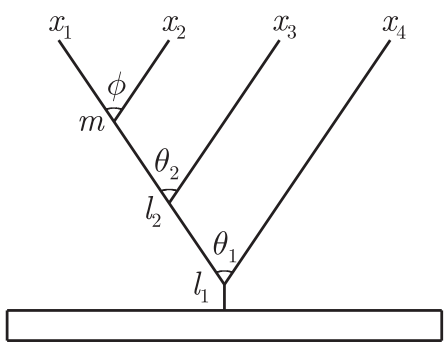

(d)

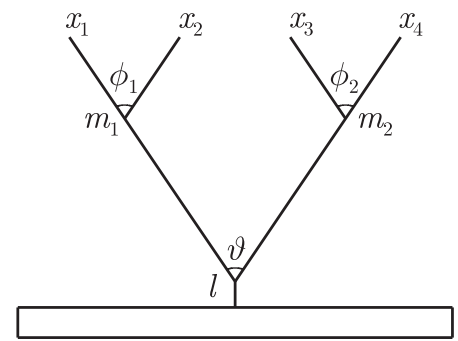

(e)

Figure 3. Tree diagrams for four dimensional polyspherical coordinate systems of type: (a) $\mathbf{b}^{2} \mathbf{a}$, (b) $\mathbf{b} \mathbf{b}^{\prime} \mathbf{a}$, (c) $\mathbf{b}^{\prime} \mathbf{b a}$, (d) $\mathbf{b}^{\prime 2} \mathbf{a}$, (e) $\mathbf{c a}^{2}$.

In $d=4$ there are five possible topological trees for polyspherical coordinates. The first tree (see Figs. 3a and 4) corresponds to the coordinate system

$$
\begin{array}{ll}
x_{1}=r \cos \theta_{1}, & x_{2}=r \sin \theta_{1} \cos \theta_{2}, \\
x_{3}=r \sin \theta_{1} \sin \theta_{2} \cos \phi, & x_{4}=r \sin \theta_{1} \sin \theta_{2} \sin \phi,
\end{array}
$$

where $\theta_{1}, \theta_{2} \in[0, \pi]$. This tree is of type $\mathbf{b}^{2} \mathbf{a}$, and the cosine of the separation angle is given by

$$
\cos \gamma=\cos \theta_{1} \cos \theta_{1}^{\prime}+\sin \theta_{1} \sin \theta_{1}^{\prime}\left(\cos \theta_{2} \cos \theta_{2}^{\prime}+\sin \theta_{2} \sin \theta_{2}^{\prime} \cos \left(\phi-\phi^{\prime}\right)\right) \text {. }
$$

The second, third and fourth trees (see Figs. 3b, 3c, 3d) are equivalent to the first.

The fifth tree (see Figs. 3e and 5b) corresponds to Hopf coordinates,

$$
\begin{array}{ll}
x_{1}=r \cos \vartheta \cos \phi_{1}, & x_{2}=r \cos \vartheta \sin \phi_{1}, \\
x_{3}=r \sin \vartheta \cos \phi_{2}, & x_{4}=r \sin \vartheta \sin \phi_{2},
\end{array}
$$

where $\vartheta \in\left[0, \frac{\pi}{2}\right]$ and $\phi_{1}, \phi_{2} \in[0,2 \pi)$. This tree is of type $\mathbf{c a}^{2}$. The cosine of the separation angle is given by

$$
\cos \gamma=\cos \vartheta \cos \vartheta^{\prime} \cos \left(\phi_{1}-\phi_{1}^{\prime}\right)+\sin \vartheta \sin \vartheta^{\prime} \cos \left(\phi_{2}-\phi_{2}^{\prime}\right) .
$$

There are many choices for polyspherical coordinates on $\mathbb{R}^{d}$, suitably defined for any number of dimensions $d \geq 2$. The simplest example of a polyspherical coordinate system on $\mathbb{R}^{d}$ (which generalizes type ba and $\mathbf{b}^{2} \mathbf{a}$ ) are what we refer to as standard polyspherical coordinates (see Fig. 4). These are

$$
\begin{aligned}
& x_{1}=r \cos \theta_{1}, \\
& x_{2}=r \sin \theta_{1} \cos \theta_{2}, \\
& x_{3}=r \sin \theta_{1} \sin \theta_{2} \cos \theta_{3}, \\
& \ldots \ldots \ldots \ldots \ldots \ldots \ldots
\end{aligned}
$$




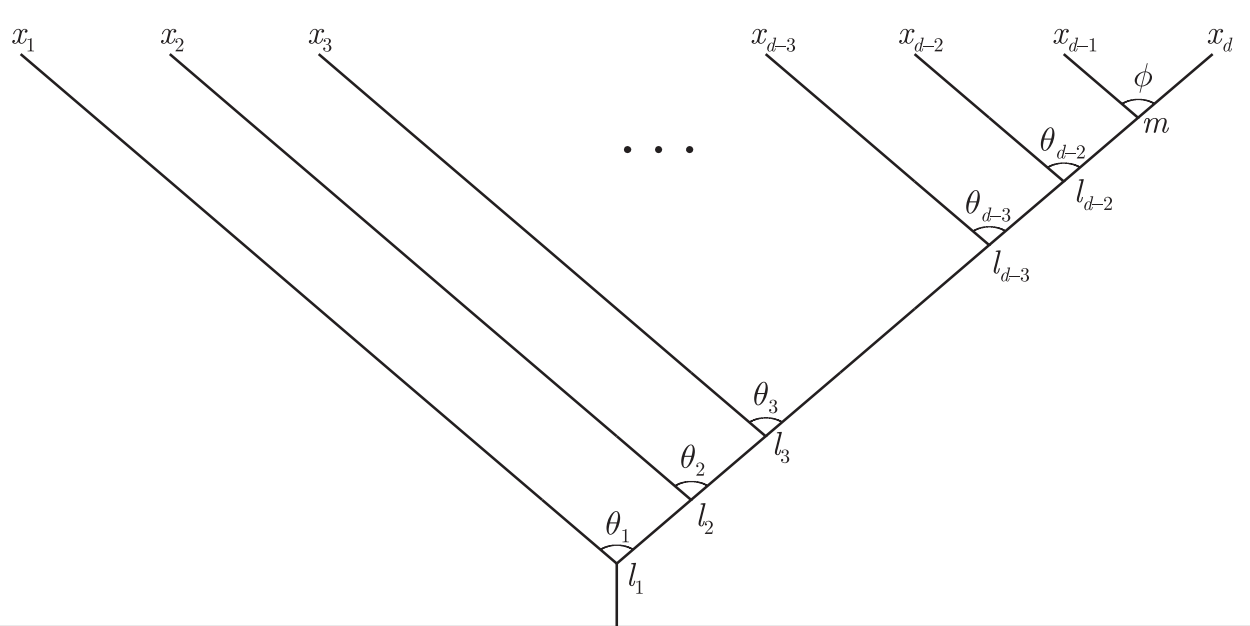

Figure 4. Tree diagram for $d$-dimensional standard polyspherical coordinates of type $\mathbf{b}^{d-2} \mathbf{a}$ (standard polyspherical coordinates).

$$
\begin{aligned}
& x_{d-2}=r \sin \theta_{1} \cdots \sin \theta_{d-3} \cos \theta_{d-2}, \\
& x_{d-1}=r \sin \theta_{1} \cdots \sin \theta_{d-3} \sin \theta_{d-2} \cos \phi, \\
& x_{d}=r \sin \theta_{1} \cdots \sin \theta_{d-3} \sin \theta_{d-2} \sin \phi,
\end{aligned}
$$

where $\theta_{i} \in[0, \pi]$ for $1 \leq i \leq d-2$ and $\phi \in[0,2 \pi)$. Using our naming procedure, this tree is of type $\mathbf{b}^{d-2} \mathbf{a}$. In these coordinates the cosine of the separation angle is given by

$$
\cos \gamma=\sum_{i=1}^{d-2} \cos \theta_{i} \cos \theta_{i}^{\prime} \prod_{j=1}^{i-1} \sin \theta_{j} \sin \theta_{j}^{\prime}+\cos \left(\phi-\phi^{\prime}\right) \prod_{i=1}^{d-2} \sin \theta_{i} \sin \theta_{i}^{\prime} .
$$

Another example of a polyspherical coordinate system which is valid for large dimensions is what we will refer to as generalized Hopf coordinates (see Fig. 5). These coordinates, valid on $\mathbb{R}^{2^{q}}$ for $q \geq 1$, generalize two-dimensional polar coordinates (B.5, type a) and four-dimensional Hopf coordinates (B.9, type $\mathbf{c a}^{2}$ ). These coordinates are unique in that they correspond to the only trees which contain only themselves in their equivalence class (see (B.2)). These coordinate systems have separated harmonic eigenfunctions which are given in terms of complex exponentials, and for $q \geq 2$, non-symmetric Jacobi polynomials (see Fig. 5).

This coordinate system is suitably defined for dimensions $d=2^{q}$ for $q \geq 1$. The transformation formulae to Cartesian coordinates are given by

$$
\begin{aligned}
& x_{1}=r \cos \vartheta_{1} \cos \vartheta_{2} \cos \vartheta_{4} \cos \vartheta_{8} \cdots \cos \vartheta_{2^{q-2}} \cos \phi_{1} \text {, } \\
& x_{2}=r \cos \vartheta_{1} \cos \vartheta_{2} \cos \vartheta_{4} \cos \vartheta_{8} \cdots \cos \vartheta_{2^{q-2}} \sin \phi_{1} \text {, } \\
& x_{2^{q-1}-1}=r \cos \vartheta_{1} \sin \vartheta_{2} \sin \vartheta_{5} \sin \vartheta_{11} \cdots \sin \vartheta_{3 \cdot 2^{q-3}-1} \cos \phi_{2^{q-2}} \text {, } \\
& x_{2^{q-1}}=r \cos \vartheta_{1} \sin \vartheta_{2} \sin \vartheta_{5} \sin \vartheta_{11} \cdots \sin \vartheta_{3 \cdot 2^{q-3}-1} \sin \phi_{2^{q-2}} \text {, } \\
& x_{2^{q-1}+1}=r \sin \vartheta_{1} \cos \vartheta_{3} \cos \vartheta_{6} \cos \vartheta_{12} \cdots \cos \vartheta_{3 \cdot 2^{q-3}} \cos \phi_{2^{q-2}+1} \text {, } \\
& x_{2^{q-1}+2}=r \sin \vartheta_{1} \cos \vartheta_{3} \cos \vartheta_{6} \cos \vartheta_{12} \cdots \cos \vartheta_{3 \cdot 2^{q-3}} \sin \phi_{2^{q-2}+1} \text {, }
\end{aligned}
$$

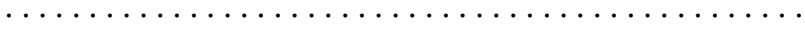

$$
\begin{aligned}
& x_{2^{q}-1}=r \sin \vartheta_{1} \sin \vartheta_{3} \sin \vartheta_{7} \sin \vartheta_{17} \cdots \sin \vartheta_{2^{q-1}-1} \cos \phi_{2^{q-1}}, \\
& x_{2^{q}}=r \sin \vartheta_{1} \sin \vartheta_{3} \sin \vartheta_{7} \sin \vartheta_{17} \cdots \sin \vartheta_{2^{q-1}-1} \sin \phi_{2^{q-1}} \text {, }
\end{aligned}
$$




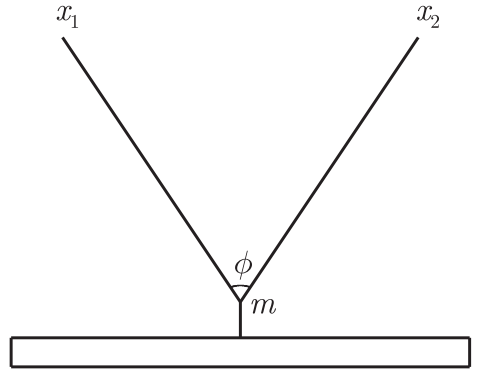

(a)

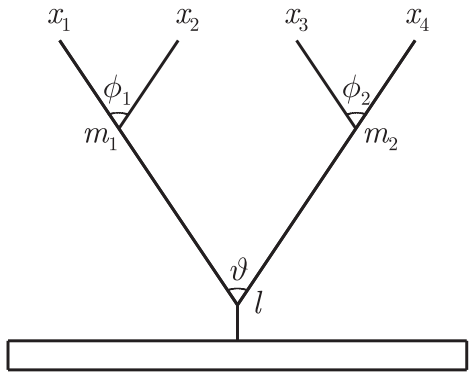

(b)

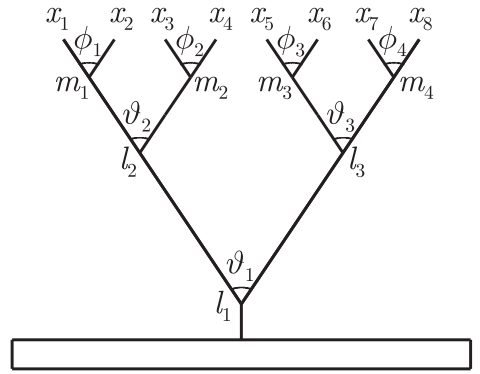

(c)

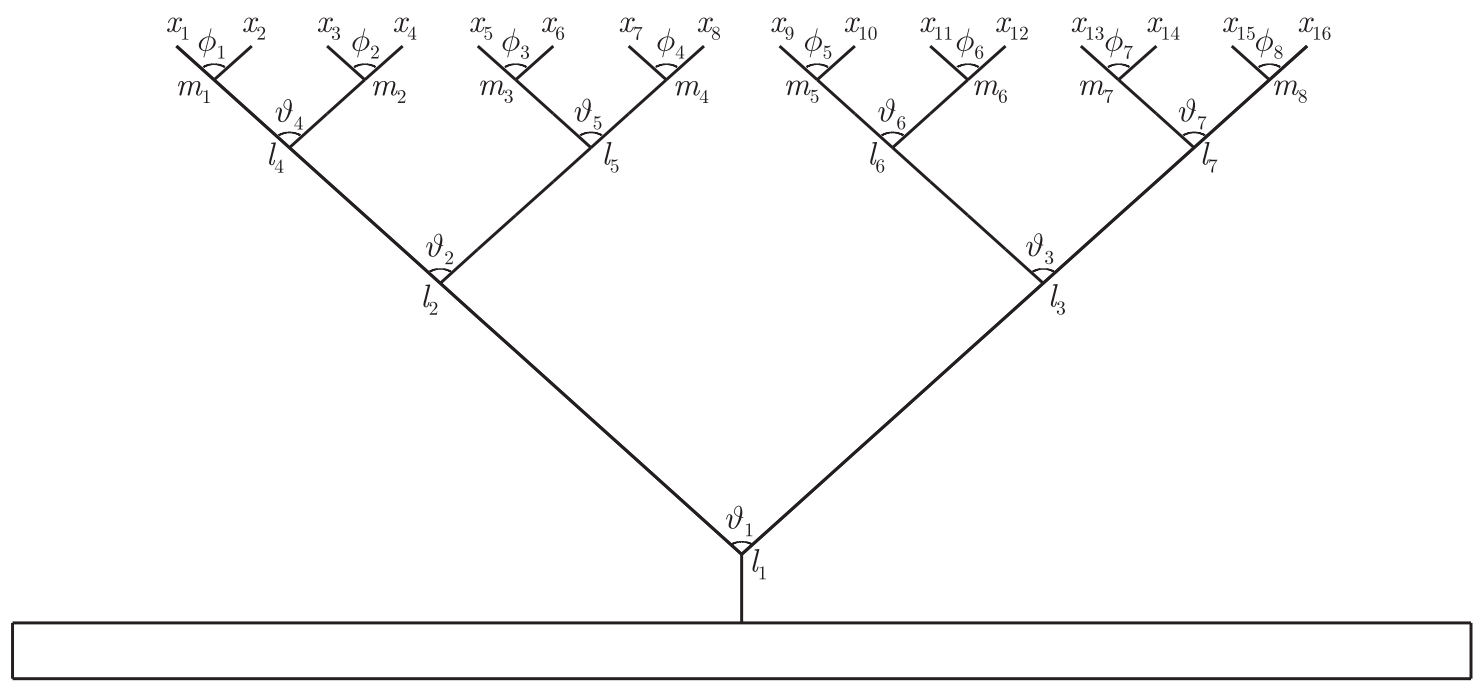

(d)

Figure 5. This figure is a tree diagram for polyspherical generalized Hopf coordinates of type $\mathbf{V}_{2^{q}}$ on $\mathbb{R}^{2^{q}}$ with $q=1,2,3,4$ for (a), (b), (c), (d) respectively. The first $\left(2^{q-1}-1\right)$-branching nodes are of type $\mathbf{c}$ which correspond to the angles $\vartheta_{i} \in\left[0, \frac{\pi}{2}\right]$ and quantum numbers $l_{i} \in \mathbb{N}_{0}$. The following $\left(2^{q}\right)$-branching nodes are of type a which correspond to the angles $\phi_{i} \in[0,2 \pi)$ and quantum numbers $m_{i} \in \mathbb{Z}$. These coordinates correspond to transformation (B.12).

where $\vartheta_{i} \in\left[0, \frac{\pi}{2}\right]$ for $1 \leq i \leq 2^{q-1}-1$ and $\phi_{i} \in[0,2 \pi)$ for $1 \leq i \leq 2^{q-1}$. Using our naming procedure, these coordinates are of type

$$
\mathbf{V}_{2^{q}}=\mathbf{c V}_{2^{q-1}} \mathbf{V}_{2^{q-1}}
$$

where $q \in \mathbb{N}$, with type $\mathbf{V}_{2}=\mathbf{a}$ (polar) coordinates on $\mathbb{R}^{2}$ (see Figs. 2a and 5a).

The cosine of the separation angle in this coordinate system may be given as follows. Define the symbol ${ }_{q} \mathrm{G}_{s}^{r} \in[-1,1]$, where $0 \leq s \leq q$ and $1 \leq r \leq 2^{q}-1$, by the recursive formula

$$
{ }_{q} \mathrm{G}_{s}^{r}=\cos \vartheta_{r-1+2^{q-s}} \cos \vartheta_{r-1+2^{q-s}}^{\prime}{ }_{q} \mathrm{G}_{s-1}^{2 r-1}+\sin \vartheta_{r-1+2^{q-s}} \sin \vartheta_{r-1+2^{q-s}}^{\prime} \mathrm{G}_{s-1}^{2 r},
$$

with ${ }_{q} \mathrm{G}_{0}^{i}=1$. Then the cosine of the separation angle is given by

$$
\cos \gamma={ }_{q} \mathrm{G}_{q}^{1}
$$

(cf. (B.6), (B.10)). Note that through the identification $\phi_{i}=\vartheta_{i-1+2^{q-1}}$, where $1 \leq i \leq 2^{q-1}$, then ${ }_{q} \mathrm{G}_{1}^{i}=\cos \left(\phi_{i}-\phi_{i}^{\prime}\right)$. Thus, this shows one may stop this recursion at $s=1$.

\section{B.2 Hyperspherical harmonics in polyspherical coordinates}

The eigenfunction expansions for a power-law fundamental solution of the polyharmonic equation in polyspherical coordinates can be derived using a Gegenbauer polynomial expansion for the 
relevant kernel (see Corollary 2) in conjunction with the addition theorem for hyperspherical harmonics. This addition theorem is given by

$$
C_{n}^{d / 2-1}(\cos \gamma)=\frac{2(d-2) \pi^{d / 2}}{(2 n+d-2) \Gamma(d / 2)} \sum_{K} Y_{n}^{K}(\widehat{\mathbf{x}}) \overline{Y_{n}^{K}\left(\widehat{\mathbf{x}}^{\prime}\right)}
$$

(for a proof see [35]; see also $\S 10.2 .1$ in [14]), where $K$ stands for a set of $(d-2$ )-quantum numbers identifying harmonics for a given value of $n \in \mathbb{N}_{0}$, and $\cos \gamma$ is the cosine of the separation angle between two arbitrary vectors $\mathbf{x}, \mathbf{x}^{\prime} \in \mathbb{R}^{d}$ (see (B.4)). The functions $Y_{n}^{K}: \mathbb{S}^{d-1} \rightarrow \mathbb{C}$ are the normalized hyperspherical harmonics. Normalization of the hyperspherical harmonics is achieved through

$$
\int_{\mathbb{S}^{d-1}} Y_{n}^{K}(\widehat{\mathbf{x}}) \overline{Y_{n}^{K}(\widehat{\mathbf{x}})} d \Omega=1
$$

where $d \Omega$ is the Riemannian volume measure on $\mathbb{S}^{d-1}$.

The general basis functions that one obtains by putting coordinates on the $d$-dimensional unit hypersphere $\mathbb{S}^{d-1}$, can be specified as solutions to the angular part of Laplace's equation on $\mathbb{R}^{d}$. These correspond to separated solutions of Laplace's equation, using the Laplace-Beltrami operator on the hypersphere $\mathbb{S}^{d-1}$. The following numbers are associated with each branching node $m \in \mathbb{Z}, l, l_{\alpha}, l_{\beta} \in \mathbb{N}_{0}$ (see Fig. 1 ). The number of vertices above each branching node $l_{\alpha}$ and $l_{\beta}$ are represented by $S_{\alpha}$ and $S_{\beta}$ respectively.

The following separated factors of eigenfunctions are generated at each branching node for normalized hyperspherical harmonics in polyspherical coordinates using the method of trees (see for instance $[19,(2.3)-(2.6)],[33, \S 10.5 .3])$ :

- Type a:

$$
\Psi_{m}\left(\phi_{\mathbf{a}}\right)=\frac{1}{\sqrt{2 \pi}} e^{i m \phi_{\mathbf{a}}}, \quad m \in \mathbb{Z}, \quad \phi_{\mathbf{a}} \in[0,2 \pi) .
$$

- Type b:

$$
\begin{aligned}
& \Psi_{n, l_{\beta}}^{\alpha}\left(\theta_{\mathbf{b}}\right)=N_{n}^{\alpha, \alpha}\left(\sin \theta_{\mathbf{b}}\right)^{l_{\beta}} P_{n}^{(\alpha, \alpha)}\left(\cos \theta_{\mathbf{b}}\right), \\
& n=l-l_{\beta}, \quad \alpha=l_{\beta}+\frac{S_{\beta}}{2}, \quad n \in \mathbb{N}_{0}, \quad \theta_{\mathbf{b}} \in[0, \pi] .
\end{aligned}
$$

- Type $\mathbf{b}^{\prime}$ :

$$
\begin{aligned}
& \Psi_{n, l_{\alpha}}^{\beta}\left(\theta_{\mathbf{b}^{\prime}}\right)=N_{n}^{\beta, \beta}\left(\cos \theta_{\mathbf{b}^{\prime}}\right)^{l_{\alpha}} P_{n}^{(\beta, \beta)}\left(\sin \theta_{\mathbf{b}^{\prime}}\right), \\
& n=l-l_{\alpha}, \quad \beta=l_{\alpha}+\frac{S_{\alpha}}{2}, \quad n \in \mathbb{N}_{0}, \quad \theta_{\mathbf{b}^{\prime}} \in\left[-\frac{\pi}{2}, \frac{\pi}{2}\right] .
\end{aligned}
$$

- Type c:

$$
\begin{aligned}
& \Psi_{n, l_{\alpha}, l_{\beta}}^{\alpha, \beta}\left(\vartheta_{\mathbf{c}}\right)=2^{(\alpha+\beta) / 2+1} N_{n}^{\alpha, \beta}\left(\sin \vartheta_{\mathbf{c}}\right)^{l_{\beta}}\left(\cos \vartheta_{\mathbf{c}}\right)^{l_{\alpha}} P_{n}^{(\beta, \alpha)}\left(\cos 2 \vartheta_{\mathbf{c}}\right), \\
& n=\frac{1}{2}\left(l-l_{\alpha}-l_{\beta}\right), \quad \alpha=l_{\alpha}+\frac{S_{\alpha}}{2}, \quad \beta=l_{\beta}+\frac{S_{\beta}}{2}, \\
& n \in \mathbb{N}_{0}, \quad \vartheta_{\mathbf{c}} \in\left[0, \frac{\pi}{2}\right] \text {. }
\end{aligned}
$$


We refer to the quantum number $n \in \mathbb{N}_{0}$ in (B.14)-(B.16) as the surrogate quantum number to $l \in \mathbb{N}_{0}$.

Notice that the eigenfunctions for branching nodes of type $\mathbf{b}$ and $\mathbf{b}^{\prime}$ can be expressed in terms of Gegenbauer polynomials using (A.7). Therefore we can re-write (B.14) as

$$
\Psi_{n, l_{\beta}}^{\alpha}\left(\theta_{\mathbf{b}}\right)=\frac{(2 \alpha) !}{\Gamma(\alpha+1)} \sqrt{\frac{(2 \alpha+2 n+1) n !}{2^{2 \alpha+1}(2 \alpha+n) !}}\left(\sin \theta_{\mathbf{b}}\right)^{l_{\beta}} C_{n}^{\alpha+1 / 2}\left(\cos \theta_{\mathbf{b}}\right),
$$

and (B.15) as

$$
\Psi_{n, l_{\alpha}}^{\beta}\left(\theta_{\mathbf{b}^{\prime}}\right)=\frac{(2 \beta) !}{\Gamma(\beta+1)} \sqrt{\frac{(2 \beta+2 n+1) n !}{2^{2 \beta+1}(2 \beta+n) !}}\left(\sin \theta_{\mathbf{b}^{\prime}}\right)^{l_{\alpha}} C_{n}^{\beta+1 / 2}\left(\cos \theta_{\mathbf{b}^{\prime}}\right) .
$$

Note that even though $\alpha, \beta$ are not necessarily integers, $2 \alpha, 2 \beta \in \mathbb{N}_{0}$.

With the simplest example, polar coordinates (see Fig. 2a, transformation (B.5)), the normalized harmonics are given by

$$
Y_{m}(\phi)=\frac{e^{i m \phi}}{\sqrt{2 \pi}}
$$

In $d=3$ there are two equivalent polyspherical coordinate systems, that of type ba (B.7) and $\mathbf{b}^{\prime} \mathbf{a}$. Since they are equivalent coordinate systems, we treat only the first. This tree has two branching nodes. Using (B.14) we see that $\alpha=m, n=l-m, l_{\beta}=m$, and $S_{\beta}=0$ since there are no vertices above the branching node $m$. Through reduction and multiplication by the $\phi$ eigenfunction, the normalized spherical harmonics are

$$
Y_{l, m}(\theta, \phi)=(-1)^{m} \sqrt{\frac{2 l+1}{4 \pi} \frac{(l-m) !}{(l+m) !}} \mathrm{P}_{l}^{m}(\cos \theta) e^{i m \phi} .
$$

Notice we have used (A.12) with (B.17) to reduce the Gegenbauer polynomial to a Ferrers function of the first kind. The functions $Y_{l, m}:[0, \pi] \times[0,2 \pi) \rightarrow \mathbb{C}$ are called standard spherical harmonics.

In $d=4$ we consider type $\mathbf{b}^{2} \mathbf{a}$ (see Fig. 3a) coordinates, whose normalized hyperspherical harmonics are

$$
\begin{aligned}
Y_{l_{1}, l_{2}, m}\left(\theta_{1}, \theta_{2}, \phi\right)= & \frac{(-1)^{m}\left(2 l_{2}\right) ! !}{\pi} \sqrt{\frac{\left(2 l_{2}+1\right)\left(l_{1}+1\right)\left(l_{1}-l_{2}\right) !\left(l_{2}-m\right) !}{2\left(l_{1}+l_{2}+1\right) !\left(l_{2}+m\right) !}} \\
& \times\left(\sin \theta_{1}\right)^{l_{2}} C_{l_{1}-l_{2}}^{l_{2}+1}\left(\cos \theta_{1}\right) \mathrm{P}_{l_{2}}^{m}\left(\cos \theta_{2}\right) e^{i m \phi} .
\end{aligned}
$$

In type $\mathbf{c a}^{2}$ (see Fig. 3e), the normalized hyperspherical harmonics are

$$
\begin{aligned}
Y_{l, m_{1}, m_{2}}\left(\vartheta, \phi_{1}, \phi_{2}\right)= & \frac{e^{i\left(m_{1} \phi_{1}+m_{2} \phi_{2}\right)}}{\pi} \sqrt{\frac{l+1}{2} \frac{\left[\frac{1}{2}\left(l+\left|m_{1}\right|+\left|m_{2}\right|\right] !\left[\frac{1}{2}\left(l-\left|m_{1}\right|-\left|m_{2}\right|\right)\right] !\right.}{\left[\frac{1}{2}\left(l-\left|m_{1}\right|+\left|m_{2}\right|\right)\right] !\left[\frac{1}{2}\left(l+\left|m_{1}\right|-\left|m_{2}\right|\right)\right] !}} \\
& \times(\sin \vartheta)^{\left|m_{2}\right|}(\cos \vartheta)^{\left|m_{1}\right|} P_{\left(l-\left|m_{1}\right|-\left|m_{2}\right|\right) / 2}^{\left(\left|m_{2}\right|,\left|m_{1}\right|\right)}(\cos 2 \vartheta),
\end{aligned}
$$

with the restriction to the parameter space given by $\frac{1}{2}\left(l-\left|m_{1}\right|-\left|m_{2}\right|\right) \in \mathbb{N}_{0}$. Using the surrogate quantum number $n \in \mathbb{N}_{0}$, defined in terms of $l \in \mathbb{N}_{0}$ such that $2 n=l-\left|m_{1}\right|-\left|m_{2}\right|$, we can more conveniently express the normalized hyperspherical harmonics in type $\mathbf{c a}^{2}$ coordinates, namely

$$
Y_{n, m_{1}, m_{2}}\left(\vartheta, \phi_{1}, \phi_{2}\right)=\frac{e^{i\left(m_{1} \phi_{1}+m_{2} \phi_{2}\right)}}{\pi} \sqrt{\frac{\left(2 n+\left|m_{1}\right|+\left|m_{2}\right|+1\right)\left(n+\left|m_{1}\right|+\left|m_{2}\right|\right) ! n !}{2\left(n+\left|m_{1}\right|\right) !\left(n+\left|m_{2}\right|\right) !}}
$$




$$
\times(\sin \vartheta)^{\left|m_{2}\right|}(\cos \vartheta)^{\left|m_{1}\right|} P_{n}^{\left(\left|m_{2}\right|,\left|m_{1}\right|\right)}(\cos 2 \vartheta) .
$$

For arbitrary dimensions, we can use standard polyspherical coordinates (B.11) to construct the normalized hyperspherical harmonics. The polyspherical harmonics corresponding to this coordinate system are basis functions for the irreducible representations of $O(d)$ (see [32]). In terms of these coordinates, the normalized hyperspherical harmonics are

$$
Y_{l}^{K}(\widehat{\mathbf{x}})=\frac{e^{i m \phi}}{\sqrt{2 \pi}} \prod_{j=1}^{d-2} \Theta_{j}^{d}\left(l_{j}, l_{j+1} ; \theta_{j}\right)
$$

where $\widehat{\mathbf{x}} \in \mathbb{S}^{d-1}, K=\left\{l_{2}, l_{3}, \ldots, l_{d-1}\right\}, l=l_{1} \geq l_{2} \geq l_{3} \geq \cdots \geq l_{d-3} \geq l_{d-2}=\ell \geq l_{d-1}=|m| \geq 0$, and $\Theta_{j}^{d}: \mathbb{N}_{0}^{2} \times[0, \pi] \rightarrow \mathbb{R}$ is defined by

$$
\begin{aligned}
\Theta_{j}^{d}\left(l_{j}, l_{j+1} ; \theta_{j}\right):= & \frac{\Gamma\left(l_{j+1}+\frac{d-j+1}{2}\right)}{2 l_{j+1}+d-j-1} \sqrt{\frac{2^{2 l_{j+1}+d-j-1}\left(2 l_{j}+d-j-1\right)\left(l_{j}-l_{j+1}\right) !}{\pi\left(l_{j}+l_{j+1}+d-j-2\right) !}} \\
& \times\left(\sin \theta_{j}\right)^{l_{j+1}} C_{l_{j}-l_{j+1}}^{l_{j+1}+(d-j-1) / 2}\left(\cos \theta_{j}\right) .
\end{aligned}
$$

The computation of (B.20) is a straightforward consequence of (B.17), and doing the proper node counting for $S_{\beta}$, in the tree depicted in Fig. 4. The normalized spherical harmonics (B.18) are the simplest example of these harmonics.

In generalized Hopf coordinates of type $\mathbf{V}_{2^{p}}$ coordinates with $p \geq 1$ (see Fig. 5), the normalized hyperspherical harmonics $Y_{l_{1}}^{K}: \mathbb{S}^{2^{p}-1} \rightarrow \mathbb{C}$ can be given more conveniently using the surrogate quantum numbers $n_{q} \in \mathbb{N}_{0}$ which are connected to $l_{q} \in \mathbb{N}_{0}$ through the relation $l_{q}=l_{\alpha}+l_{\beta}+2 n_{q}$ such that $1 \leq q \leq 2^{p-1}-1$. The orthonormal polyspherical harmonics, which can be generated using the method of trees, are

$$
Y_{l_{1}}^{K}(\widehat{\mathbf{x}})=\frac{\left.e^{i\left(m_{1} \phi_{1}+\cdots+m_{2^{p-1}} \phi_{2} p-1\right.}\right)}{\sqrt{2} \pi^{2^{p-2}}} \prod_{j=1}^{p-1} \prod_{s=1}^{2^{j-1}} \Upsilon_{q}^{p}\left(\begin{array}{c}
n_{q} \\
l_{\alpha}, l_{\beta}
\end{array} ; \vartheta_{q}\right)
$$

where $K=\left\{l_{2}, \ldots, l_{2^{p-1}-1}, m_{1}, \ldots, m_{2^{p-1}}\right\}$ and $\Upsilon_{q}^{p}: \mathbb{N}_{0}^{3} \times\left[0, \frac{\pi}{2}\right] \rightarrow \mathbb{R}$ is defined by

$$
\Upsilon_{q}^{p}\left(\begin{array}{c}
n_{q} \\
l_{\alpha}, l_{\beta}
\end{array} ; \vartheta_{q}\right):=\sqrt{\frac{\left(2 n_{q}+\alpha+\beta+1\right)\left(n_{q}+\alpha+\beta\right) ! n_{q} !}{\left(n_{q}+\alpha\right) !\left(n_{q}+\beta\right) !}}\left(\cos \vartheta_{q}\right)^{l_{\alpha}}\left(\sin \vartheta_{q}\right)^{l_{\beta}} P_{n_{q}}^{(\beta, \alpha)}\left(\cos \left(2 \vartheta_{q}\right)\right)
$$

with $q=2^{j-1}+s-1, l_{\alpha}=l_{2 q}, l_{\beta}=l_{2 q+1}, \alpha=l_{2 q}-1+2^{p-2-\left\lfloor\log _{2} q\right\rfloor}$, and $\beta=l_{2 q+1}-1+$ $2^{p-2-\left\lfloor\log _{2} q\right\rfloor}$. Note that we use the identification

$$
\left(l_{2^{p-1}}, \ldots, l_{2^{p}-1}\right)=\left(\left|m_{1}\right|, \ldots,\left|m_{2^{p-1}}\right|\right) .
$$

\section{Acknowledgements}

I would like to thank A.F.M. Tom ter Elst and Heather Macbeth for valuable discussions. I would like to express my gratitude to the anonymous referees and an editor at SIGMA whose helpful comments improved this paper. Part of this work was conducted while H.S. Cohl was a National Research Council Research Postdoctoral Associate in the Applied and Computational Mathematics Division at the National Institute of Standards and Technology, Gaithersburg, Maryland, USA. 


\section{References}

[1] Abramowitz M., Stegun I.A., Handbook of mathematical functions with formulas, graphs, and mathematical tables, National Bureau of Standards Applied Mathematics Series, Vol. 55, U.S. Government Printing Office, Washington, D.C., 1964.

[2] Alonso Izquierdo A., Fuertes W.G., de la Torre Mayado M., Guilarte J.M., One-loop corrections to the mass of self-dual semi-local planar topological solitons, Nuclear Phys. B 797 (2008), 431-463, arXiv:0707.4592.

[3] Andrews G.E., Askey R., Roy R., Special functions, Encyclopedia of Mathematics and its Applications, Vol. 71, Cambridge University Press, Cambridge, 1999.

[4] Boyling J.B., Green's functions for polynomials in the Laplacian, Z. Angew. Math. Phys. 47 (1996), 485-492.

[5] Cohl H.S., Erratum: Developments in determining the gravitational potential using toroidal functions, Astronom. Nachr. 333 (2012), 784-785.

[6] Cohl H.S., Fourier and Gegenbauer expansions for fundamental solutions of the Laplacian and powers in $\mathbb{R}^{d}$ and $\mathbb{H}^{d}$, Ph.D. thesis, The University of Auckland, Auckland, New Zealand, 2010.

[7] Cohl H.S., Fourier expansions for a logarithmic fundamental solution of the polyharmonic equation, arXiv:1202.1811.

[8] Cohl H.S., Dominici D.E., Generalized Heine's identity for complex Fourier series of binomials, Proc. R. Soc. Lond. Ser. A Math. Phys. Eng. Sci. 467 (2011), 333-345, arXiv:0912.0126.

[9] Cohl H.S., Kalnins E.G., Fourier and Gegenbauer expansions for a fundamental solution of the Laplacian in the hyperboloid model of hyperbolic geometry, J. Phys. A: Math. Theor. 45 (2012), 145206, 32 pages, arXiv:1105.0386.

[10] Cohl H.S., Rau A.R.P., Tohline J.E., Browne D.A., Cazes J.E., Barnes E.I., Useful alternative to the multipole expansion of $1 / r$ potentials, Phys. Rev. A 64 (2001), 052509, 5 pages, arXiv:1104.1499.

[11] Cohl H.S., Tohline J.E., Rau A.R.P., Srivastava H.M., Developments in determining the gravitational potential using toroidal functions, Astronom. Nachr. 321 (2000), 363-372.

[12] Cormen T.H., Leiserson C.E., Rivest R.L., Stein C., Introduction to algorithms, 2nd ed., MIT Press, Cambridge, MA, 2001.

[13] Erdélyi A., Magnus W., Oberhettinger F., Tricomi F.G., Higher transcendental functions, Vol. II, McGrawHill, New York - Toronto - London, 1953.

[14] Fano U., Rau A.R.P., Symmetries in quantum physics, Academic Press Inc., San Diego, CA, 1996.

[15] Gel'fand I.M., Shilov G.E., Generalized functions. Vol. I. Properties and operations, Academic Press, New York, 1964.

[16] Gradshteyn I.S., Ryzhik I.M., Table of integrals, series, and products, 7th ed., Elsevier/Academic Press, Amsterdam, 2007.

[17] Ismail M.E.H., Classical and quantum orthogonal polynomials in one variable, Encyclopedia of Mathematics and its Applications, Vol. 98, Cambridge University Press, Cambridge, 2005.

[18] Izmest'ev A.A., Pogosyan G.S., Sissakian A.N., Winternitz P., Contractions of Lie algebras and separation of variables. The $n$-dimensional sphere, J. Math. Phys. 40 (1999), 1549-1573.

[19] Izmest'ev A.A., Pogosyan G.S., Sissakian A.N., Winternitz P., Contractions of Lie algebras and the separation of variables: interbase expansions, J. Phys. A: Math. Gen. 34 (2001), 521-554.

[20] Kil'dyushov M.S., Hyperspherical functions of the "tree" type in the $n$-body problem, Soviet J. Nuclear Phys. 15 (1972), 113-118.

[21] Koekoek J., Koekoek R., The Jacobi inversion formula, Complex Variables Theory Appl. 39 (1999), 1-18, math.CA/9908148.

[22] Lake M., Thomas S., Ward J., Non-topological cycloops, J. Cosmol. Astropart. Phys. 2010 (2010), no. 1, 026, 27 pages, arXiv:0911.3118.

[23] Lin C.D., Hyperspherical coordinate approach to atomic and other Coulombic three-body systems, Phys. Rep. 257 (1995), 1-83.

[24] Lin F., Yang Y., Energy splitting, substantial inequality, and minimization for the Faddeev and Skyrme models, Comm. Math. Phys. 269 (2007), 137-152.

[25] Magnus W., Oberhettinger F., Soni R.P., Formulas and theorems for the special functions of mathematical physics, 3rd ed., Die Grundlehren der mathematischen Wissenschaften, Bd. 52, Springer-Verlag, New York, 1966. 
[26] Miller Jr. W., Symmetry and separation of variables, Encyclopedia of Mathematics and its Applications, Vol. 4, Addison-Wesley Publishing Co., Reading, Mass. - London - Amsterdam, 1977.

[27] Olver F.W.J., Lozier D.W., Boisvert R.F., Clark C.W. (Editors), NIST handbook of mathematical functions, U.S. Department of Commerce National Institute of Standards and Technology, Washington, DC, 2010.

[28] Schwartz L., Théorie des distributions. Tome I, Actualités Sci. Ind., no. 1091, Hermann \& Cie., Paris, 1950.

[29] Sloane N.J.A., Plouffe S., The encyclopedia of integer sequences, Academic Press Inc., San Diego, CA, 1995.

[30] Stanley R.P., Enumerative combinatorics. Vol. 2, Cambridge Studies in Advanced Mathematics, Vol. 62, Cambridge University Press, Cambridge, 1999.

[31] Szegő G., Orthogonal polynomials, American Mathematical Society Colloquium Publications, Vol. 23, Amer. Math. Soc., Providence, R.I., 1959.

[32] Vilenkin N.Ja., Special functions and the theory of group representations, Translations of Mathematical Monographs, Vol. 22, Amer. Math. Soc., Providence, R.I., 1968.

[33] Vilenkin N.Ja., Klimyk A.U., Representation of Lie groups and special functions. Vol. 2. Class I representations, special functions, and integral transforms, Mathematics and its Applications (Soviet Series), Vol. 74, Kluwer Academic Publishers Group, Dordrecht, 1993.

[34] Vilenkin N.Ja., Kuznetsov G.I., Smorodinskiǔ Ya.A., Eigenfunctions of the Laplace operator providing representations of the $\mathrm{U}(2), \mathrm{SU}(2), \mathrm{SO}(3), \mathrm{U}(3)$ and $\mathrm{SU}(3)$ groups and the symbolic method, Soviet J. Nuclear Phys. 2 (1965), 645-652.

[35] Wen Z.Y., Avery J., Some properties of hyperspherical harmonics, J. Math. Phys. 26 (1985), 396-403. 